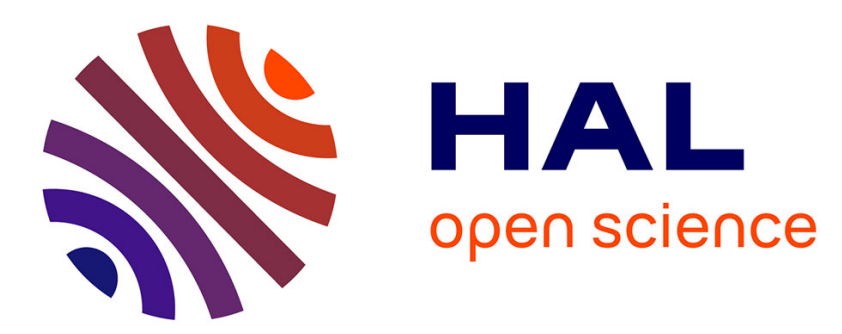

\title{
Numerical modelling of negative discharges in air with experimental validation
}

\author{
T N Tran, I O Golosnoy, P L Lewin, G E Georghiou
}

\section{To cite this version:}

T N Tran, I O Golosnoy, P L Lewin, G E Georghiou. Numerical modelling of negative discharges in air with experimental validation. Journal of Physics D: Applied Physics, 2011, 44 (1), pp.15203. 10.1088/0022-3727/44/1/015203 . hal-00597849

\section{HAL Id: hal-00597849 \\ https://hal.science/hal-00597849}

Submitted on 2 Jun 2011

HAL is a multi-disciplinary open access archive for the deposit and dissemination of scientific research documents, whether they are published or not. The documents may come from teaching and research institutions in France or abroad, or from public or private research centers.
L'archive ouverte pluridisciplinaire HAL, est destinée au dépôt et à la diffusion de documents scientifiques de niveau recherche, publiés ou non, émanant des établissements d'enseignement et de recherche français ou étrangers, des laboratoires publics ou privés. 


\title{
Numerical modelling of negative discharges in air with experimental validation
}

\author{
T N Tran ${ }^{1}$, I O Golosnoy ${ }^{1}$, P L Lewin ${ }^{1}$ and G E Georghiou ${ }^{2}$ \\ ${ }^{1)}$ School of Electronics and Computer Science, University of Southampton, \\ Southampton SO17 1BJ, UK \\ ${ }^{2)}$ Department of Electrical and Computer Engineering, University of Cyprus, \\ 1678 Nicosia, Cyprus \\ Email: i.golosnoy@soton.ac.uk
}

\begin{abstract}
Axisymmetric finite element models have been developed for the simulation of negative discharges in air without and with presence of dielectrics. The models are based on the hydrodynamic drift-diffusion approximation. A set of continuity equations accounting for the movement, generation and loss of charge carriers (electrons, positive and negative ions) are coupled with Poisson's equation to take into account the effect of space and surface charges on the electric field. The model of a negative corona discharge (without dielectric barriers) in a needle- -plane geometry is analysed first. The results obtained show good agreement with experimental observations for various Trichel pulse characteristics. With dielectric barriers introduced into the discharge system, the surface discharge exhibits some similarities and differences to the corona case. The model studies the dynamics of volume charge generation, electric field variations and charge accumulation over the dielectric surface. The predicted surface charge density is consistent with experimental results obtained from the Pockels experiment in terms of distribution form and magnitude.
\end{abstract}

Keywords: corona discharge, surface discharge, finite element simulation, needle plane, Trichel pulses.

\section{Introduction}

Non-thermal plasmas produced from gas discharges have received a lot of attention in recent years due to their broad applications and practical issues [1,2]. In the absence of a dielectric, corona discharges have been utilised for the treatment of gaseous pollutants, surface treatment, lighting and electrostatic painting $[1,3,4]$. Corona discharges can also lead to serious problems such as arcs in RF processing or power losses in transmission lines [5]. In the presence of a dielectric(s), the discharges are often regarded as dielectric barrier discharges (DBD) or silent discharges. At atmospheric pressure, DBDs have found their applications in large-scale ozone production, plasma display panels, thin film deposition, actuators for flow control, $\mathrm{CO}_{2}$ lasers and excimer ultra-violet light sources $[1,6]$. Additionally surface charge accumulation at triple-junctions in gas-insulated switchgear leading to breakdown of insulation system has also been a topic of research for a while [7].

Depending on the polarity of the applied voltage, the plasma structure varies from filamentary to diffuse patterns. The latter is produced from a negative applied voltage and is often preferred for certain applications due to its stability, uniformity and efficiency [1]. The negative corona discharge has been well studied experimentally and the most distinctive feature is often regarded to as Trichel pulses [8]. Numerical modelling work on this subject has mostly been limited to 1D-1.5D models [9-11]. Regarding dielectric barrier discharge, 
most simulation work have concentrated on the positive discharge in a needle- -plane geometry while attention on the negative polarity is not as extensive. Thus the aim of this paper is to develop 2D axisymmetric finite element models for the negative corona and barrier discharges and validate the model parameters by comparing with experimental data. The hydrodynamic drift-diffusion approach is utilised which consists of coupled continuity equations for the charge transport and Poisson's equation for the electric field.

\section{Experimental studies}

Figure 1 shows the schematic diagram of the experimental setup used to measure the Trichel current pulses in air at atmospheric pressure. A needle electrode of $250 \mu \mathrm{m}$ radius of curvature was utilised and connected to a negative dc high voltage supply. The needle was placed $3.3 \mathrm{~mm}$ away from the plane electrode which is made of stainless steel and has a radius of $10 \mathrm{~cm}$ and thickness of $5 \mathrm{~mm}$. The plane electrode was grounded via a shielded low inductance $75 \Omega$ shunt resistor. The voltage across the shunt resistor is transmitted to a digital oscilloscope via a $75 \Omega$ impedance co-axial cable and terminated using a $75 \Omega$ termination to eliminate reflections. The entire experiment was housed in a Faraday cage to minimise external interferences.

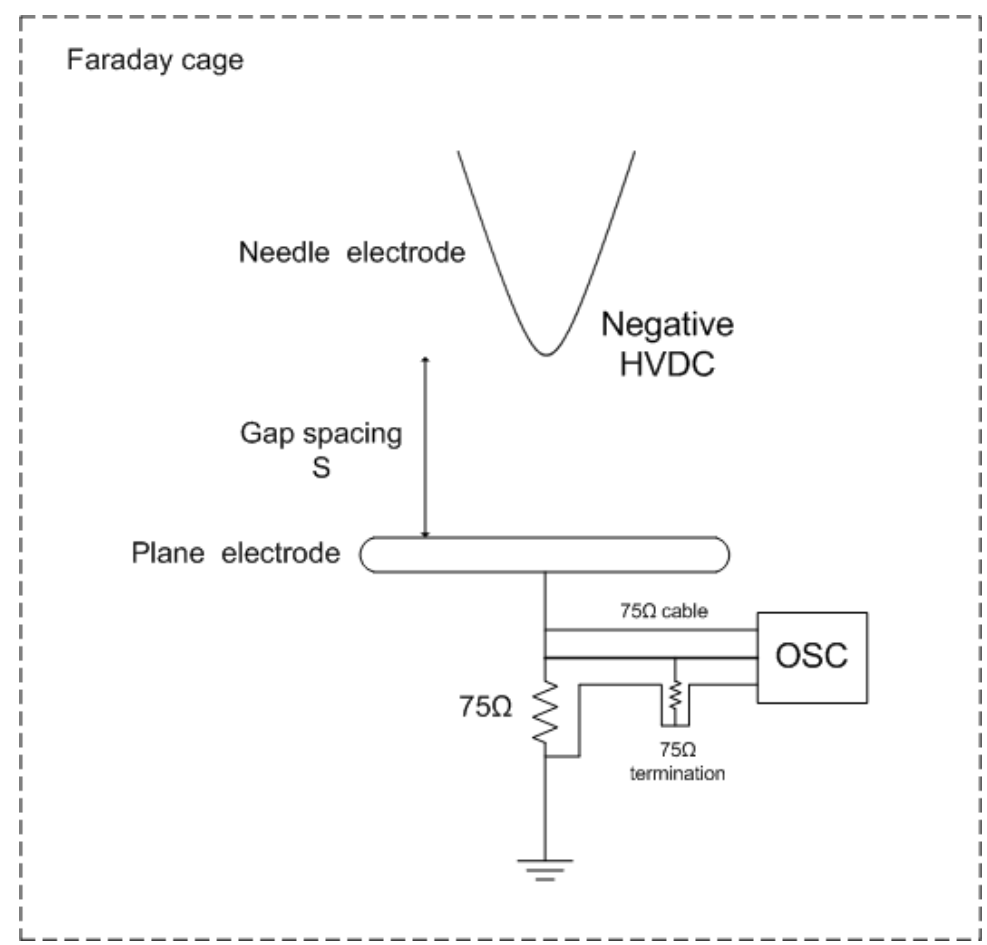

Figure 1. Schematic diagram of the negative corona discharge experiment.

With regard to measurements of surface charge accumulation over solid dielectrics, various techniques have been developed over the years including dust figures, Lichtenberg figures, electrostatic probes and the Pockels technique [7, 12]. Out of these methods, the electro-optic Pockels technique is the only method that offers quantitative analysis of surface discharge dynamics. The schematic diagram of the Pockels experiment is shown in figure 2. 


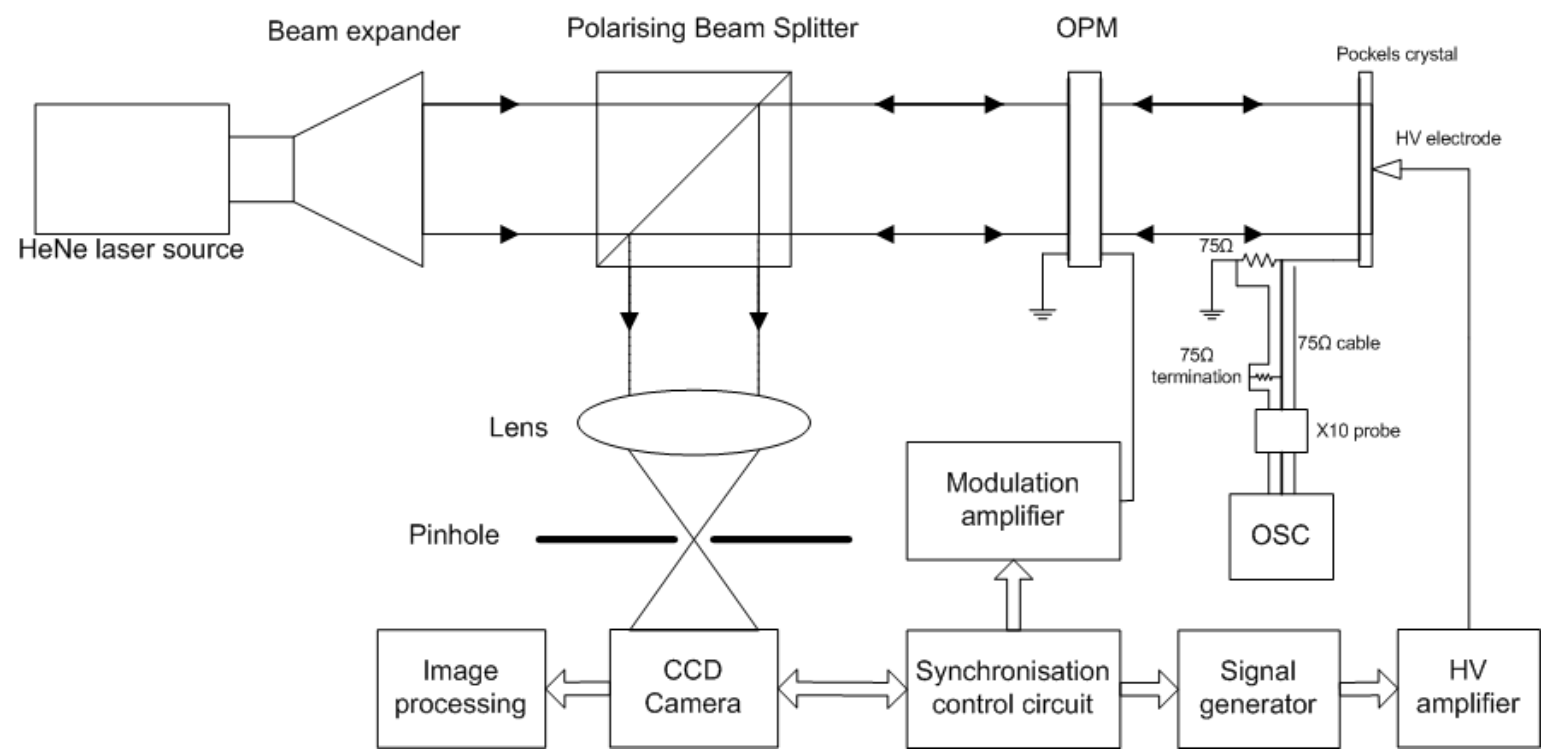

Figure 2. Schematic diagram of the Pockels experiment.

The principle of the method is based on the Pockels characteristic of some materials such as Bisthmuth Silicon Oxide (BSO). Under the electric field caused by surface charge, the refractive indexes of the BSO crystal along two perpendicular principal axes (so-called fast and slow axes) are altered. The change in the refractive indexes will lead to a linear phase retardation of the incident light. The change in phase is linearly proportional to the internal electric field. By measuring the light intensity of the reflected light, the phase retardation can be determined and hence the surface charge density. Further details of the experiment can be found in [12]. This method has been used to measure surface charge density produced by applying a negative square voltage waveform to a $25 \mu \mathrm{m}$ radius of curvature needle electrode placed $500 \mu \mathrm{m}$ away from the Pockels cell.

\section{Model description}

\subsection{Governing equations}

Based on the well-known hydrodynamic drift-diffusion model [1], the set of continuity equations (to account for the movement, generation and loss of electrons, positive and negative ions and for the development of the space charge) coupled with Poisson's equation (to include the effect of space charge on the electric field) is given as

$$
\begin{gathered}
\frac{\partial N_{e}}{\partial t}=N_{e} \alpha\left|\overrightarrow{W_{e}}\right|-N_{e} \eta\left|\overrightarrow{W_{e}}\right|-N_{e} N_{p} \beta_{e p}-\nabla \cdot\left(N_{e} \overrightarrow{W_{e}}-D_{e} \nabla N_{e}\right) \\
\frac{\partial N_{p}}{\partial t}=N_{e} \alpha\left|\overrightarrow{W_{e}}\right|-N_{n} N_{p} \beta_{n p}-N_{e} N_{p} \beta_{e p}-\nabla \cdot\left(N_{p} \overrightarrow{W_{p}}-D_{p} \nabla N_{p}\right) \\
\frac{\partial N_{n}}{\partial t}=N_{e} \eta\left|\overrightarrow{W_{e}}\right|-N_{n} N_{p} \beta_{n p}-\nabla \cdot\left(N_{n} \overrightarrow{W_{n}}-D_{n} \nabla N_{n}\right) \\
\nabla \cdot\left(\varepsilon_{r} \nabla V\right)+\frac{e}{\varepsilon_{0}}\left(N_{p}-N_{e}-N_{n}\right)=0
\end{gathered}
$$

where $t$ is time, $e$ the electronic charge, $\varepsilon_{0}$ the vacuum permittivity and $\varepsilon_{r}$ the relative permittivity of the medium, $V$ the electric potential; $N_{e}, N_{p}$ and $N_{n}$ the electron, positive and negative ion concentrations respectively; $\overrightarrow{W_{e}}, \overrightarrow{W_{p}}$ and $\overrightarrow{W_{n}}$ the electron, positive and negative 
ion drift velocities; $\alpha, \eta, \beta, D_{e}, D_{p}$ and $D_{n}$ the ionisation, attachment, recombination and diffusion coefficients for electrons and positive and negative ions respectively. The swarm parameters, which are functions of the local electric field, $\vec{E}=-\nabla V$, were extracted from the approximations published in [13] and summarised in [1] with an addition of the recombination coefficient equal to $2 \times 10^{-13}\left(\mathrm{~m}^{3} . \mathrm{s}^{-1}\right)$ as commonly used in the literature [1].

The plasma kinetic model is simplified in equations 1-4; only three sorts of charged particles: electrons, positive and abstract negative ions are taken into account. This model is, however, adequate as shown by many previous publications (see references in the review [1]). Particularly in the case of negative ions, their density is at least one order of magnitude lower compared to the positive ion and electron densities [14]. The complex ionisation, attachment and recombination kinetics were hence not considered as this would add considerably to the computational burden without affecting the results significantly. If other cases where the negative ions have a significant contribution to the development of the discharge were to be considered then the issue of complex plasma kinetics should be included (discharges in $\mathrm{SF}_{6}$, for example).

In the presence of a dielectric, zero current through the barrier and charge accumulation at the surface were assumed. The surface charge density can be calculated by integrating the normal component of charged particle fluxes at the surface as expressed in equation 5

$$
\sigma_{s}(t)=\int_{0}^{t}\left(\left.\vec{J}_{e}\right|_{\text {norm }}+\left.\vec{J}_{n}\right|_{\text {norm }}+\left.\vec{J}_{p}\right|_{\text {norm }}\right) d t \text { or } \frac{\partial \sigma_{s}(t)}{\partial t}=\left.\vec{J}_{e}\right|_{\text {norm }}+\left.\vec{J}_{n}\right|_{\text {norm }}+\left.\vec{J}_{p}\right|_{\text {norm }}
$$

where the charge fluxes through the surface $\left.\vec{J}_{i}\right|_{n o r m}=\left.e_{i} N_{i} \vec{W}_{i}\right|_{\text {norm }}, i=\overline{e, p, n}$. After being deposited on the surface, the charge is to be stationary; any charge motion over the dielectric is neglected.

It has long been established that secondary emission processes are the main mechanism sustaining negative discharges $[8,9]$. These electron emission processes at the cathode include the Fowler-Norheim process (electrons emitted due to a high electric field at the cathode), photo-emission (electrons produced by photons generated in discharge plasma) and ion-impact emission (electrons released as positive ions strike the cathode surface). Previous studies $[11,15]$ argue that ion-impact is the key mechanism sustaining the discharge. For this reason, the only secondary process that has been taken into account in this work is the ionimpact. By using the expression given in equation 6 , this process may represent others by varying the ion-impact secondary emission coefficient, $\gamma$.

$$
\text { input flux of electrons }\left.\equiv N_{e} \vec{W}_{e}\right|_{\text {norm }}=\left.\gamma N_{p} \overrightarrow{W_{p}}\right|_{\text {norm }}
$$

Different values of $\gamma$ have been used in the literature and they often fall in the range from $10^{-3}$ to $10^{-2}[9-11,15-16]$.

\subsection{Computational domain}

The simulation geometries utilised in this work are shown in figure 3. In the negative corona discharge model, a hyperboloid-shaped needle electrode was assumed of $250 \mu \mathrm{m}$ radius of curvature. The distance between the needle and the ground electrode was set at $3.3 \mathrm{~mm}$. These were to match with the dimensions of the experimental setup. In the negative surface discharge model, a system of two dielectrics was modelled to resemble the Pockels cell. A $160 \mu \mathrm{m}$ thick BSO layer $\left(\varepsilon_{r}=56\right)$ was set on top of a $800 \mu \mathrm{m}$ thick glass $\left(\varepsilon_{r}=3\right)$. Both layers share the same radius of $10 \mathrm{~mm}$. The BSO surface was set $500 \mu \mathrm{m}$ away from the needle electrode. A different electrode of $25 \mu \mathrm{m}$ radius of curvature was used to facilitate a higher electric field. 


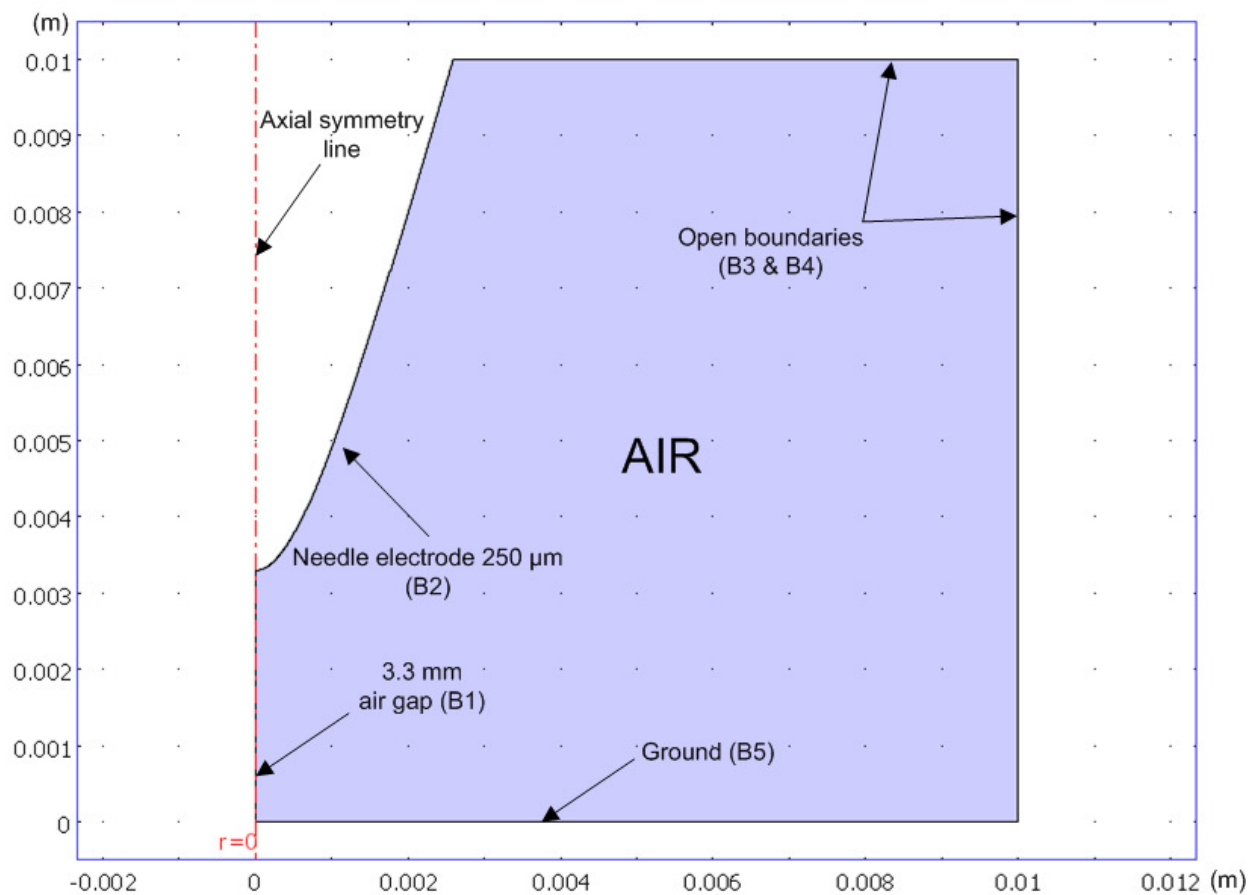

(a) Negative corona discharge

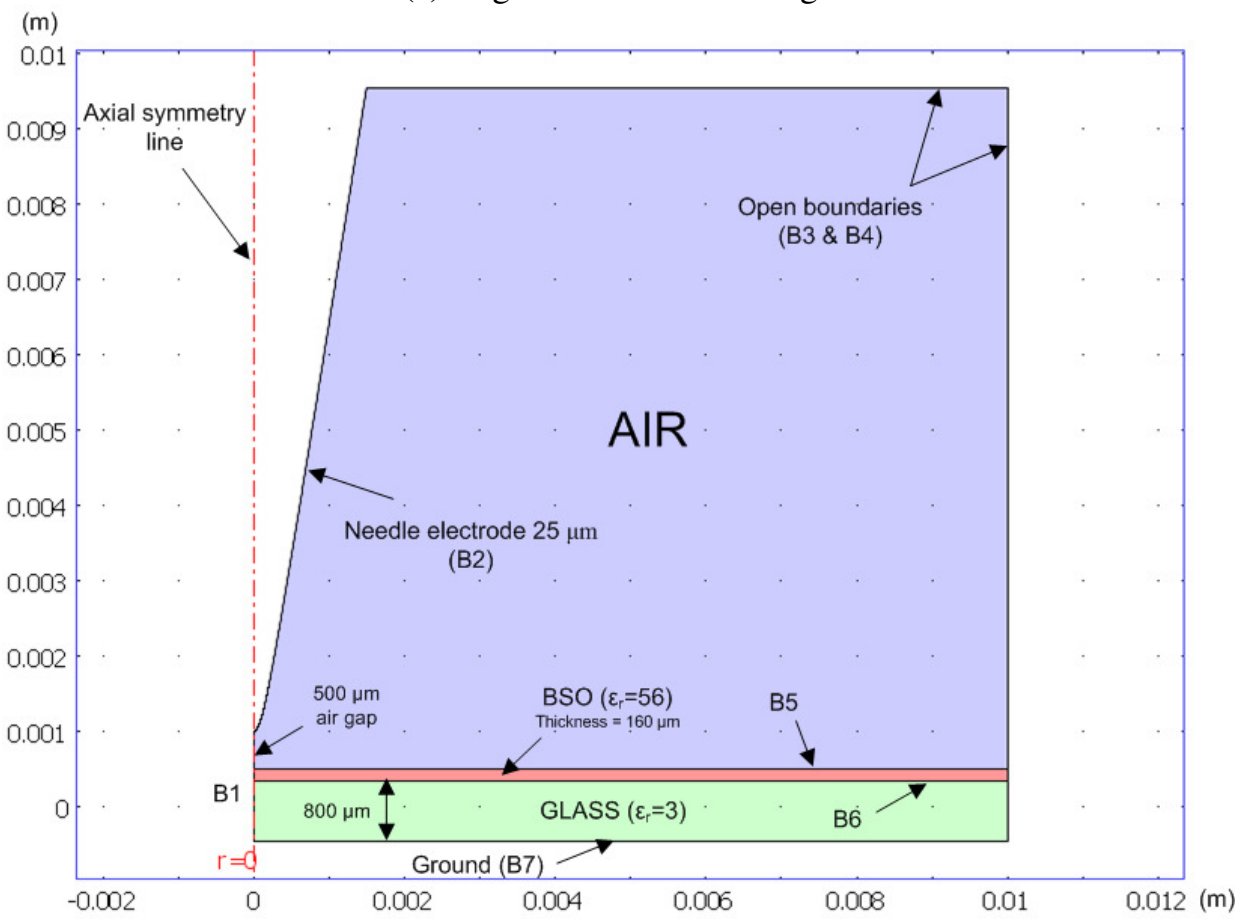

(b) Negative dielectric barrier discharge

Figure 3. Simulation geometry of the negative discharge models.

\subsection{Discharge parameters}

In order to compute the discharge current, two different methods have been utilised. The first approach is based on the energy conservation law which was detailed in [17] where the discharge current, $I_{V}$, is calculated in a similar manner to [18]. 


$$
I_{V}=\frac{1}{V_{a}}\left(\frac{d}{d t} \int_{V} Q d V+\int_{V} P_{D} d V\right)
$$

where $V_{a}$ is the external applied voltage, $P_{D}=\vec{E} \cdot \sum_{e, p, n} e_{i} N_{i} \vec{W}_{i}$ and $Q=\frac{1}{2} \varepsilon_{0} \varepsilon_{r}|\vec{E}|^{2}$.

Equation 7 is an extension of the well known Sato's expression [19] to time-dependent voltage [18]. Two expressions are detailed in [18]; the first one uses the total field as equation 7 whereas the second one utilises the Laplacian field only. Here it is more convenient to use the first expression since all data required is readily obtained from the model. The other method to calculate the current is to investigate the charge transport across domain boundaries. The electric field is calculated in quasi-static approximation in the model. The fixed applied potential requires surface charges to be built on the electrode surfaces. The time variation of the total surface charge at the cathode is supplied by the external circuit (current $I_{S}$ ) or by the plasma fluxes, $J$ terms in equation 8 . The current is hence calculated by integrating the charged particle fluxes through the cathode surface plus the changes of the induced surface charge at the cathode.

$$
I_{S}=\underset{\text { cathode }}{ }\left[\left(\left.\vec{J}_{e}\right|_{\text {norm }}+\left.\vec{J}_{n}\right|_{\text {norm }}+\left.\vec{J}_{p}\right|_{\text {norm }}\right) \cdot d \vec{S}+\frac{d}{d t}\left(\sigma_{c a} \cdot \vec{n} \cdot d \vec{S}\right)\right]
$$

where the needle surface charge density is computed from Gauss' theorem $\sigma_{c a}=-\vec{n} \cdot\left(\varepsilon_{0} \varepsilon_{r}\left(\overrightarrow{E_{c}}-\overrightarrow{E_{m}}\right)\right)\left(\overrightarrow{E_{c}}\right.$ and $\overrightarrow{E_{m}}$ are the electric field vectors in the plasma over the surface and inside the cathode respectively, $\overrightarrow{E_{m}}=0$ is assumed due to metal electrode). It is worth noting that the current calculated at the ground electrode via equation 8 is consistent with that calculated from the cathode surface. This is as expected since no charges should be accumulated in the external power source.

\subsection{Details of numerical algorithm}

The Convection and Diffusion application mode in COMSOL was utilised to solve the continuity equations [20]. Streamline artificial diffusion (anisotropic) was added to avoid oscillations and overshoots. COMSOL facilitates various forms of artificial diffusion when solving convection and diffusion problems [21, 22]. Isotropic diffusion introduces numerical viscosity in all directions, streamline diffusion is in the direction of drift velocity only and crosswind diffusion is orthogonal to the streamline direction [21]. Detailed comparisons of different artificial diffusion types have been presented in [22]. It has been confirmed that streamline anisotropic diffusion gives relatively good results and reasonable run time efficiency on the test problems. Crosswind diffusion provides better accuracy for the test systems but runs 10 times slower. The streamline anisotropic diffusion has proved effective to solve discharge problems in gas and liquid [17, 23] although other techniques for transport problems can be used [24].

In addition, the Electrostatics mode was employed to solve Poisson's equation. The surface charge density variation over time, equation 5, was calculated by the integration of an additional differential equation. The time derivative in equations 5,7 and 8 is linked with the time variation of the plasma electric field. The variable-order variable-step-size backward differentiation formulas (BDF) are used by COMSOL for time differentiation. (BDF is a further development of the multistep methods [25].) It makes the current calculations consistent with time discretisation of the transport equations 1-3. Space integration is performed using the standard Gaussian quadrature $\left(4^{\text {th }}\right.$ order) built in COMSOL [20]. Integration is undertaken on the same mesh used for the convection and diffusion problem.

Time steps in this model are automatically determined by COMSOL. The choice of time step is driven by the specified relative (0.01) and absolute (0.001) tolerances. The tolerances control the error in each integration step in a way that the step is accepted if the estimated local errors satisfy both tolerances [20]. The time increment is chosen automatically 
according to the algorithm summarised in [25]. As a result, the time steps vary around $10^{-10} \mathrm{~s}$ during the pulse formation and increase to $\sim 10^{-9} \mathrm{~s}$ during the decay stage. Different tolerances have been tested by comparing the solutions with that obtained using fixed time steps $\left(10^{-12}\right.$ s). It was found that the relative tolerance values of 0.01 and 0.001 (absolute tolerances were set 10 times smaller in both cases) give similar solutions (5\% difference) to that calculated from fixed time steps. The use of larger relative $(0.1)$ and absolute $(0.01)$ tolerances resulted in a $20 \%$ difference hence was discarded.

\section{Negative corona discharge}

Experimental studies on this subject revealed a well-known phenomenon known as Trichel pulses [26]. This phenomenon occurs when a negative corona discharge takes place in an electronegative gas and the measured discharge current shows a regular pulse train. Important Trichel pulse parameters include the individual pulse shape, their magnitude and frequency of occurrence. Regarding the modelling side of the phenomenon, most of the work have been undertaken in 1D-1.5D (continuity equations are solved in 1D along the symmetry axis while Poisson's equation is solved using the effectively 2D disc method) [9-11, 15]. Reports on full 2D simulations are rather limited [27]. Thus the objectives of this work are to enhance the understanding of the negative corona discharge phenomenon by the full 2D axisymmetric model, to get the model input parameters validated by experimental data and to set a benchmark to further proceed with the negative surface discharge model.

\subsection{Boundary and initial conditions}

The simulation geometry of this model is shown in figure 1(a) and the corresponding boundary conditions are summarised in table 1 .

Table 1. Boundary conditions for the negative corona discharge model.

\begin{tabular}{|c|c|c|c|c|}
\hline $\begin{array}{l}\text { Application } \\
\text { mode }\end{array}$ & $\begin{array}{c}\text { Convection and } \\
\text { diffusion } \\
N_{e}\end{array}$ & $\begin{array}{c}\text { Convection and } \\
\text { diffusion } \\
N_{p}\end{array}$ & $\begin{array}{c}\text { Convection and } \\
\text { diffusion } \\
N_{n}\end{array}$ & $\begin{array}{c}\text { Electrostatics } \\
\mathrm{V}\end{array}$ \\
\hline $\begin{array}{c}\text { Boundary } \\
1\end{array}$ & $\begin{array}{l}\text { Axial symmetry } \\
\frac{\partial N_{e}}{\partial r}=0\end{array}$ & $\begin{array}{l}\text { Axial symmetry } \\
\qquad \frac{\partial N_{p}}{\partial r}=0\end{array}$ & $\begin{array}{l}\text { Axial symmetry } \\
\qquad \frac{\partial N_{n}}{\partial r}=0\end{array}$ & $\begin{array}{l}\text { Axial symmetry } \\
\qquad \frac{\partial V}{\partial r}=0\end{array}$ \\
\hline $\begin{array}{c}\text { Boundary } \\
2\end{array}$ & $\begin{array}{c}\text { Total flux } \\
\begin{array}{c}-\vec{n} \cdot\left(\overrightarrow{W_{e}} N_{e}-D_{e} \nabla N_{e}\right)= \\
=\gamma N_{p}\left|\overrightarrow{W_{p}}\right|\end{array}\end{array}$ & $\begin{array}{l}\text { Convective flux } \\
\vec{n} \cdot\left(-D_{p} \nabla N_{p}\right)=0\end{array}$ & $\begin{array}{c}\text { Concentration } \\
N_{n}=0\end{array}$ & $\begin{array}{c}\text { Potential } \\
V=-5500(\mathrm{~V})\end{array}$ \\
\hline $\begin{array}{c}\text { Boundaries } \\
3 \& 4\end{array}$ & $\begin{array}{l}\text { Convective flux } \\
\vec{n} \cdot\left(-D_{e} \nabla N_{e}\right)=0\end{array}$ & $\begin{array}{l}\text { Convective flux } \\
\vec{n} \cdot\left(-D_{p} \nabla N_{p}\right)=0\end{array}$ & $\begin{array}{l}\text { Convective flux } \\
\vec{n} \cdot\left(-D_{n} \nabla N_{n}\right)=0\end{array}$ & $\begin{array}{l}\text { Zero charge/ } \\
\text { Symmetry } \\
\vec{n} \cdot\left(\varepsilon_{0} \varepsilon_{r} \vec{E}\right)=0\end{array}$ \\
\hline $\begin{array}{c}\text { Boundary } \\
5\end{array}$ & $\begin{array}{l}\text { Convective flux } \\
\vec{n} \cdot\left(-D_{e} \nabla N_{e}\right)=0\end{array}$ & $\begin{array}{c}\text { Concentration } \\
\qquad N_{p}=0\end{array}$ & $\begin{array}{l}\text { Convective flux } \\
\vec{n} \cdot\left(-D_{n} \nabla N_{n}\right)=0\end{array}$ & $\begin{array}{c}\text { Ground } \\
V=0\end{array}$ \\
\hline
\end{tabular}

In this model, a step voltage of $-5.5 \mathrm{kV}$ was applied to the needle. A Gaussian distribution of neutral plasma (seed electrons and positive ions) with a peak value of $10^{16} \mathrm{~m}^{-3}$ and width of $25 \mu \mathrm{m}$ was set at the needle tip according to the following equation [28]

$$
N_{e, p}=N_{\max } \times \exp \left(-\frac{\left(r-r_{0}\right)^{2}}{2 s_{0}^{2}}-\frac{\left(z-z_{0}\right)^{2}}{2 s_{0}^{2}}\right)
$$


where $N_{\max }=10^{16} \mathrm{~m}^{-3}, r_{0}=0 \mu \mathrm{m}, z_{0}$ corresponds to the needle tip position and $s_{0}=25 \mu \mathrm{m}$. The initial condition has been confirmed to only speed up the pulse formation and not to change the discharge characteristics.

The secondary emission coefficient $\gamma$ was set to 0.004 ; as will be shown later this value gives the best agreement with experiment. During the development of the model, it was realised that major discharge activities occur in the region close to the cathode. For this reason, meshing around this region proves very important. A fine mesh of $1 \mu \mathrm{m}$ in size was created in the volume $100 \mu \mathrm{m}$ away from the needle axially and radially. Further away from the ionisation region, a coarser mesh was used. A total number of one million degrees of freedom was solved for the process time duration of $30 \mu \mathrm{s}$.

\subsection{Simulation results}

Details of the first discharge current pulse and of the pulse train are shown in figure 4 . The current pulses have a magnitude of around $2 \mathrm{~mA}$ and frequency of occurrence of $142 \mathrm{kHz}$. It is interesting to note that the first current pulse magnitude is not larger than the subsequent ones as opposed to that observed when the code was used for a shorter gap [28]. This can be explained by the fact that for a longer gas gap, the frequency of occurrence is lower hence space charge particles have sufficient time to clear away from the gap [26]. As a result, the subsequent current pulses occur with similar conditions to the first one. The model suggests that the first pulse only has a single peak as opposed to double peaks claimed by other simulation work $[9,15]$. The rise time of the first pulse is around $10 \mathrm{~ns}$ and the pulse falls to its 50\% peak value in approximately $30 \mathrm{~ns}$. These values have also been reported in [27]. It can be seen that the two current calculation methods give very similar results. Volume integration takes longer to calculate as compared to surface integration. In the following sections, the method of surface integration was used to compute the results for that reason.

Different stages (I, II, III and IV) during the formation of the first Trichel pulse are marked in figure 4(a) and are discussed below. To support the analyses, the axial distribution of electron, positive and negative ion and space charge densities are plotted in figure 5. In addition, 2D snapshots of the distribution of charged particle density are shown in figure 6 at critical times. The variation of the electric field over time is shown in figure 7 along the symmetry axis and in figure 8 as a $2 \mathrm{D}$ distribution.

\section{Electron multiplication (from I to II: 0-300 ns)}

During this phase the seed electrons move away from the cathode under the influence of the electric field. As they travel, electrons gain high velocity and create additional electrons and positive ions through impact ionisation. The highest concentration of positive ions and electrons are found near the needle (figure 5 - $300 \mathrm{~ns}$ curve) where the electric field is strong. As the electrons travel further away from the needle, attachment process becomes dominant in the low electric field region. This leads to a lower concentration of electrons and an increase of negative ion density over time in this region. An overall positive space charge occupies the volume near the needle $(0.35 \mathrm{~mm})$ while a slightly negative space charge fills the rest of the gap, figure 5(d). Since the created positive ions strike the surface of the needle, secondary electrons are released according to equation 6 . This cloud of electrons then repeats the same cycle as the previous one hence the charged particle densities keep increasing. Evidently, it is this secondary process that sustains the negative discharge. The typical 2D charge density snapshot at $300 \mathrm{~ns}$ in figure 6(a) clearly demonstrates that positive ions are sandwiched between electrons and the cathode. The initial applied electric field is not heavily distorted during this phase until point II, figures 7 and 8 , due to the low order of charge density $\left(\sim 10^{17} \mathrm{~m}^{-3}\right)$. 


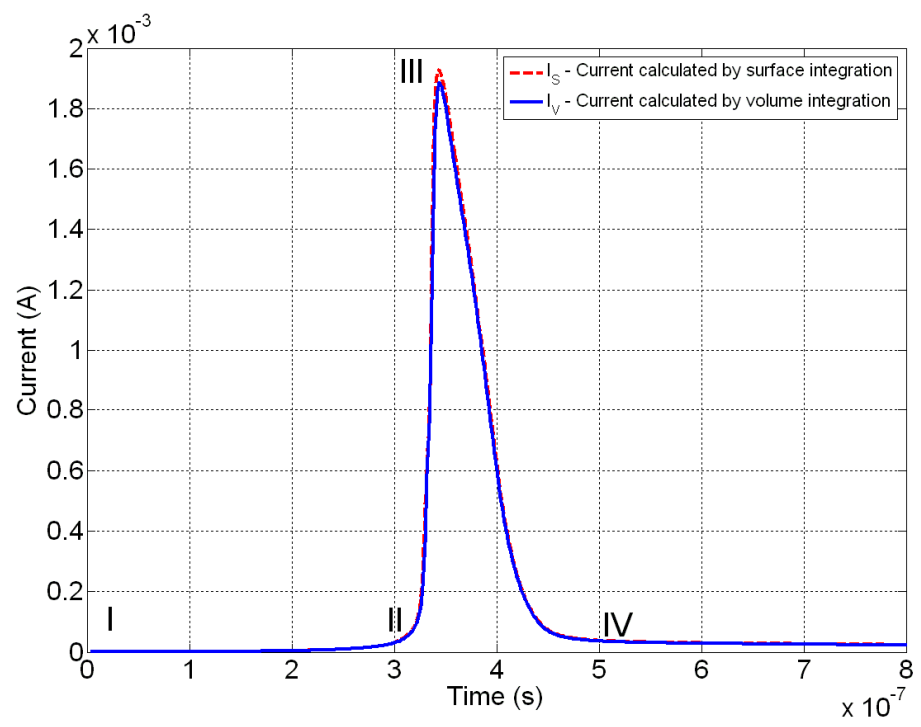

(a)

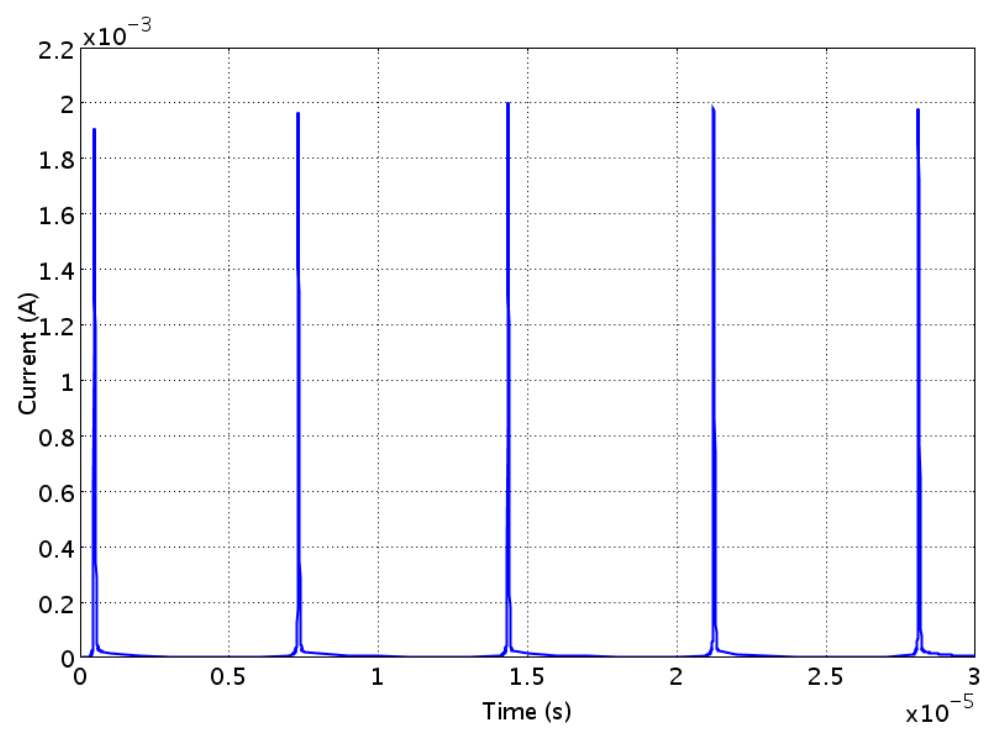

(b)

Figure 4. Predicted discharge current waveform of (a) the first Trichel pulse and (b)

Trichel pulse sequence.

Plasma sheath formation (from II to III: $300-348$ ns)

At this phase, the formation of positive ions and electrons has reached the critical concentration (order of $10^{20} \mathrm{~m}^{-3}$ ) where the space charge field strongly distorts the initial applied field. The formation of a dense plasma near the cathode increases the field on the cathode while decreases the field within the plasma (figures 7 and 8 at $330 \mathrm{~ns}$ and $348 \mathrm{~ns}$ ). The movement of charged particles during this phase can be clearly observed in figure 5 . Until $348 \mathrm{~ns}$, the peaks of both positive ion and electron densities increase and move towards the cathode. This process occurs as a result of the electric field enhancement at the needle by the plasma and has been experimentally identified as the motion of an ionising wave towards the cathode [29]. The negative ion density in this phase increases accordingly however it is still one order less than the positive counterpart. The axial space charge density plot, figure 5(d), clearly demonstrates an abrupt rise in concentration and fast movement of the charge particles during this phase. The positive space charge region is now reduced to $0.05 \mathrm{~mm}$ away from the needle followed by an increase of the negative charge concentration 
up to a distance of $0.3 \mathrm{~mm}$. The dense plasma formation can also be observed in the $2 \mathrm{D}$ charge density distribution, figure 6(b). At $348 \mathrm{~ns}$ the peak concentration of positive ions moves closest to the cathode (approximately $10 \mu \mathrm{m}$ away) and reaches its maximum density $\left(2.6 \times 10^{20} \mathrm{~m}^{-3}\right)$. This time instance also corresponds to the peak of the current waveform (point III) where the volume of positive ion concentration is reduced to its minimum. This creates a thin sheath on the cathode. High gradients of concentrations require application of a very fine mesh near the needle. It is also interesting to note that the peaks of both positive ion and electron densities are not on the symmetry axis but situated $50 \mu \mathrm{m}$ away from the axis, and dense plasma actually forms a charged torus ring rather than a disk. At $348 \mathrm{~ns}$, the electric field at the needle is approximately 6 times higher than the initial applied field whereas the field within the plasma reaches almost zero (figure 7). The ionisation region (where ionisation dominates attachment) is hence greatly reduced.

Decay process (from III to IV: 348-500 ns)

It occurs as soon as the plasma sheath formation is complete. The ionisation region has been reduced to its minimum and it is unable to sustain further discharge. The positive ion and electron densities start to drop while the negative ion density starts increasing due to attachment (figure 5, curves 450 and $500 \mathrm{~ns}$ ). At $500 \mathrm{~ns}$, the negative ion density is even higher than the electron density and of the same order as the positive charge density, figure 5(c). The space charge plot shows a decrease in magnitude of charge densities in this phase, figure $5(\mathrm{~d})$. The volume occupied by positive space charge, having shrunk in the previous phase, now expands out to $0.1 \mathrm{~mm}$ axially. During this phase the electric field tends to return to the initial field distribution (curve $500 \mathrm{~ns}$, figures 7 and 8). The next Trichel pulse occurs after the space charge particles have been gradually cleared away from the cathode by the electric field. 


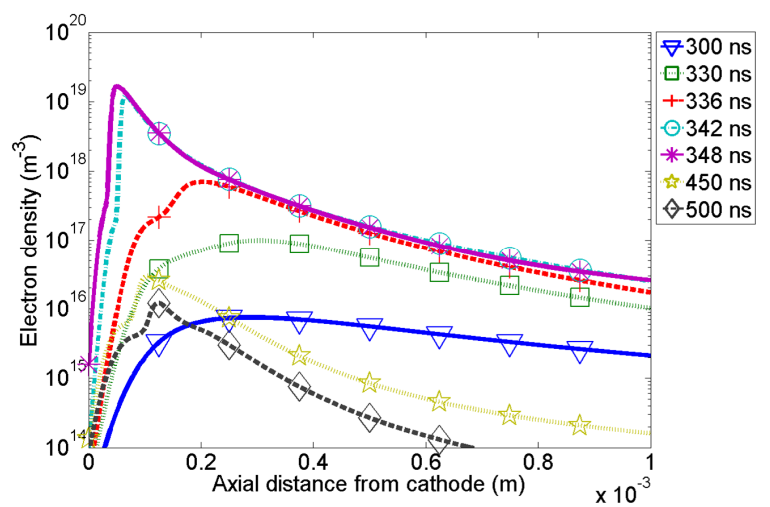

(a) Electron density distribution along the symmetry axis

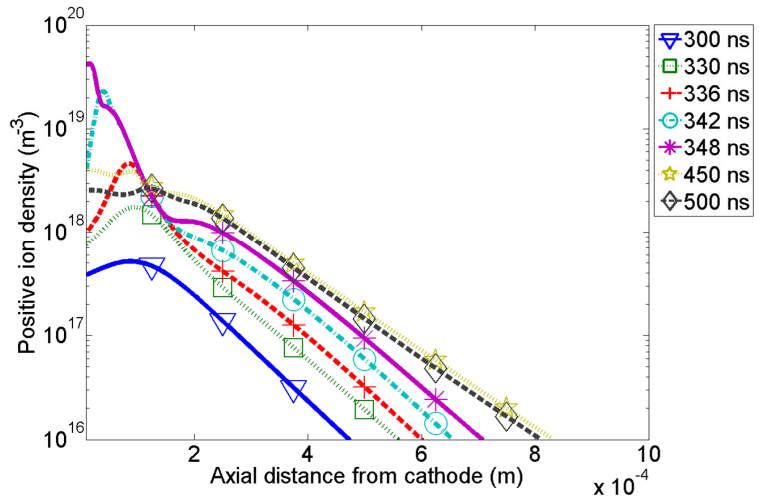

(b) Positive ion density distribution along the symmetry axis

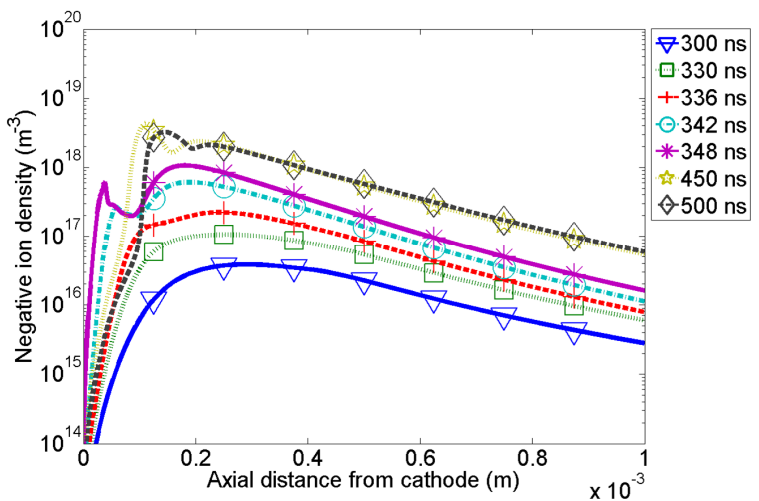

(c) Negative ion density distribution along the symmetry axis

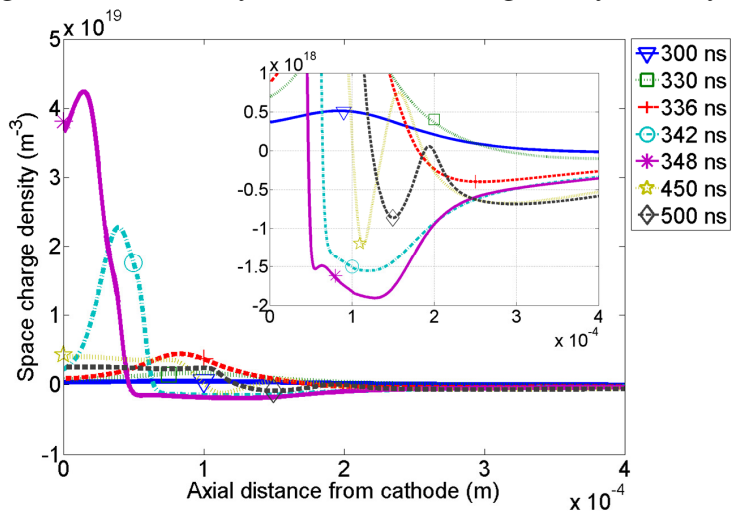

(d) Space charge density distribution along the symmetry axis

Figure 5. Predicted distributions of charged particle densities along the symmetry axis. 


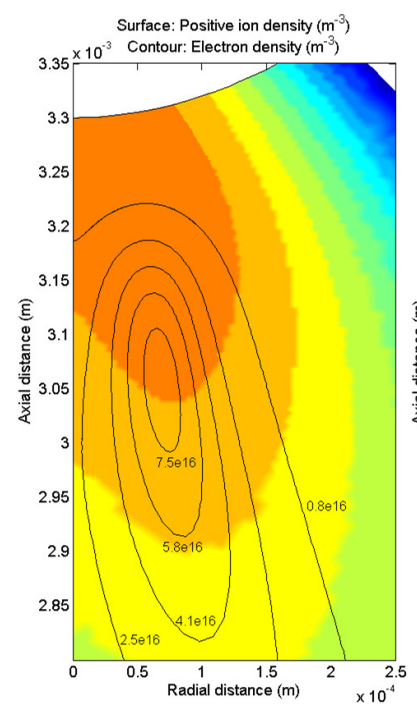

(a) $300 \mathrm{~ns}$ (II)

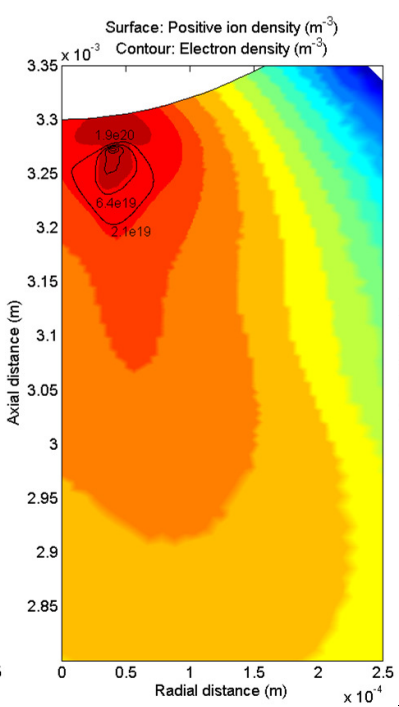

(b) $348 \mathrm{~ns}$ (III)

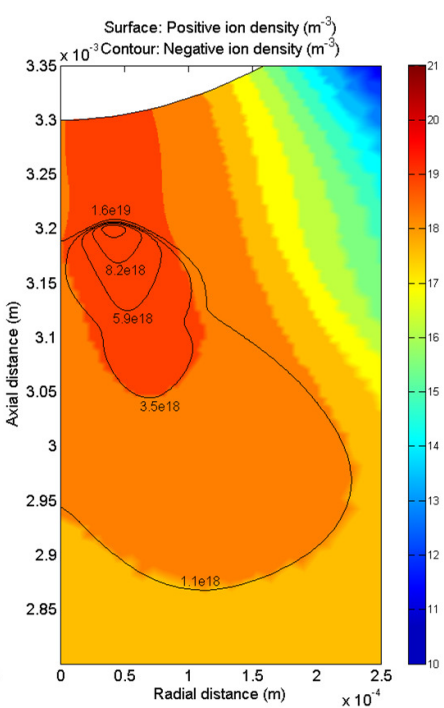

(c) $500 \mathrm{~ns}$ (IV)

Figure 6. Predicted 2D distribution of charged particles densities at critical times. Surface plots are in log scale and contour plots are in linear scale.

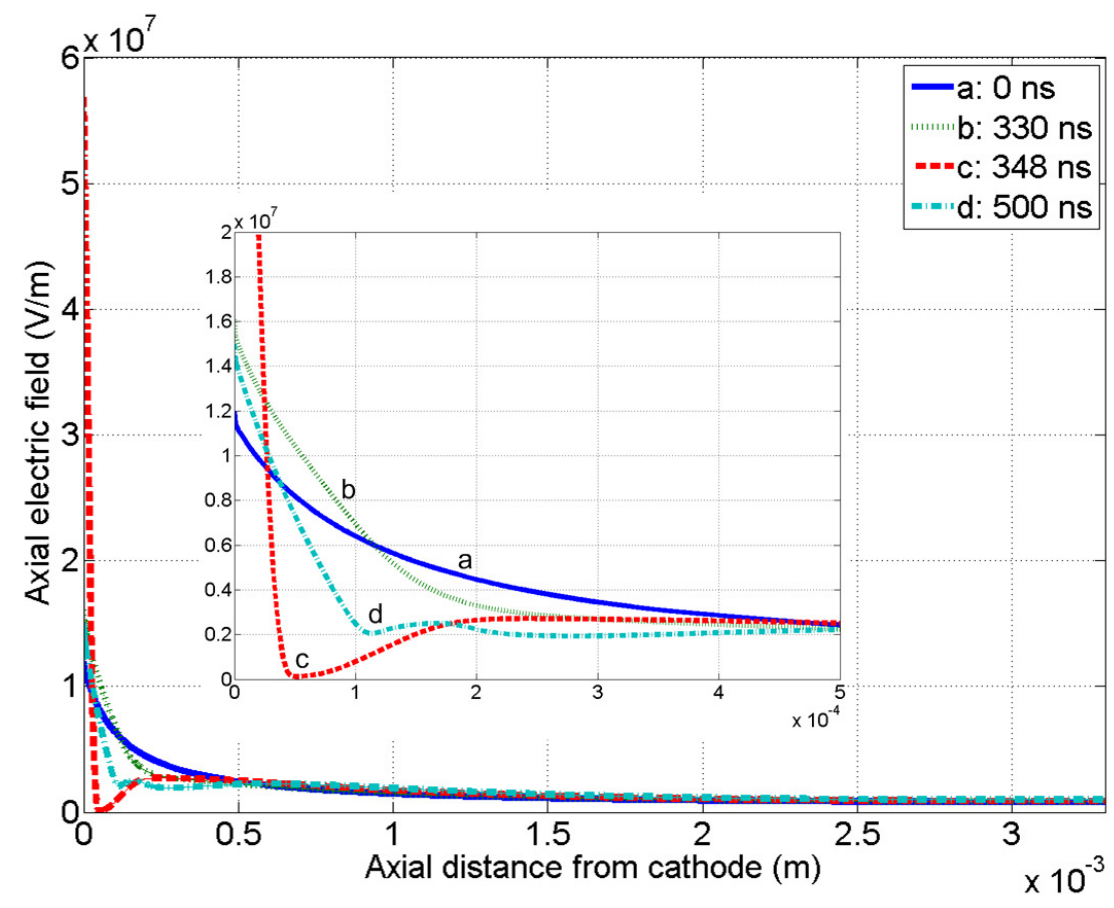

Figure 7. Predicted electric field variations with time along the symmetry axis. 


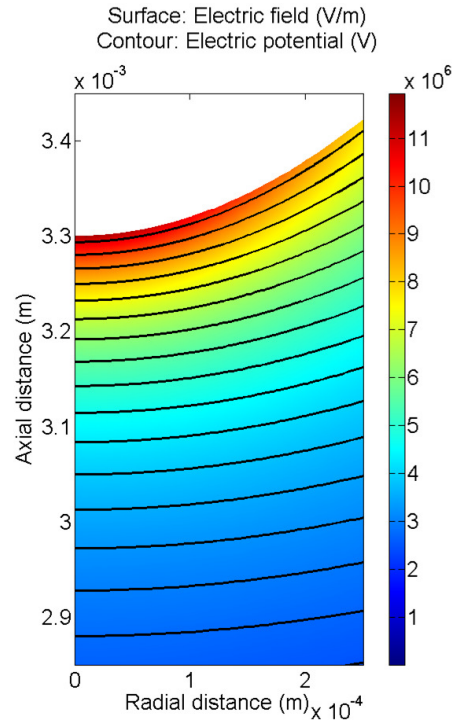

(a) $0 \mathrm{~ns}$ (I)

Surface: Electric field $(\mathrm{V} / \mathrm{m})$

Contour: Electric potential (V)

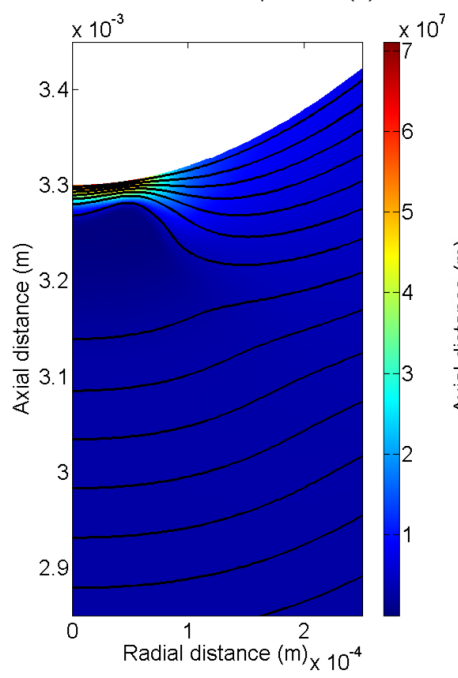

(c) $348 \mathrm{~ns}$ (III)

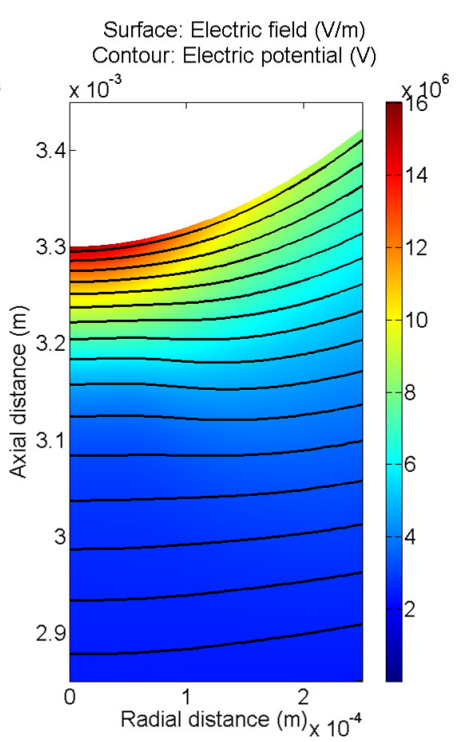

(b) $330 \mathrm{~ns}$ (II)

Surface: Electric field $(\mathrm{V} / \mathrm{m})$ Contour: Electric potential (V)

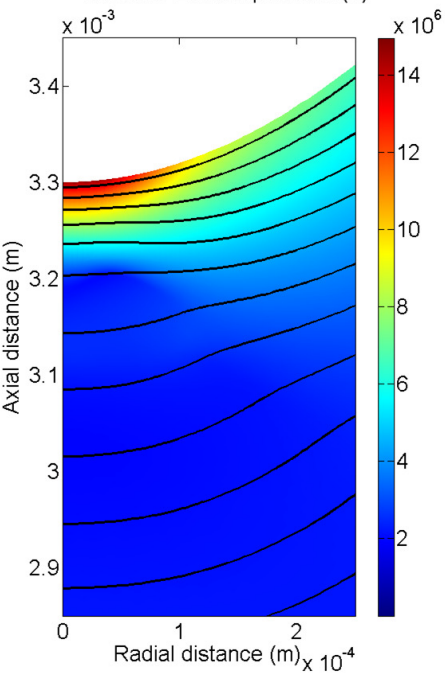

(b) $500 \mathrm{~ns}$ (IV)

Figure 8. Predicted 2D electric field distribution at critical times.

\section{Influence of the ion-impact secondary emission coefficient}

In the negative corona discharge model, the ion-impact secondary emission coefficient is the only parameter that is tuneable assuming the swarm parameters for air is well established. Effects of $\gamma$ on the formation of Trichel pulses can be studied by varying the coefficient while keeping all other parameters unchanged as specified in the model. The first current pulse magnitude and the frequency of occurrence were calculated and plotted as a function of $\gamma$ values (figure 9). As can be observed, the first Trichel pulse peak value gradually increases from 1.86 to $1.98 \mathrm{~mA}$ as $\gamma$ increases from 0.002 to 0.006 . Similarly, the frequency increases approximately linearly from $70 \mathrm{kHz}$ to $195 \mathrm{kHz}$. The averaged charge per pulse has also been calculated for each $\gamma$ value and it was confirmed that the charge also increases linearly from 0.09 to $0.12 \mathrm{nC}$ over this range of $\gamma$. These effects can be explained by noting that an increase of $\gamma$ leads to a more intensive electron production and more frequent discharge activities. 


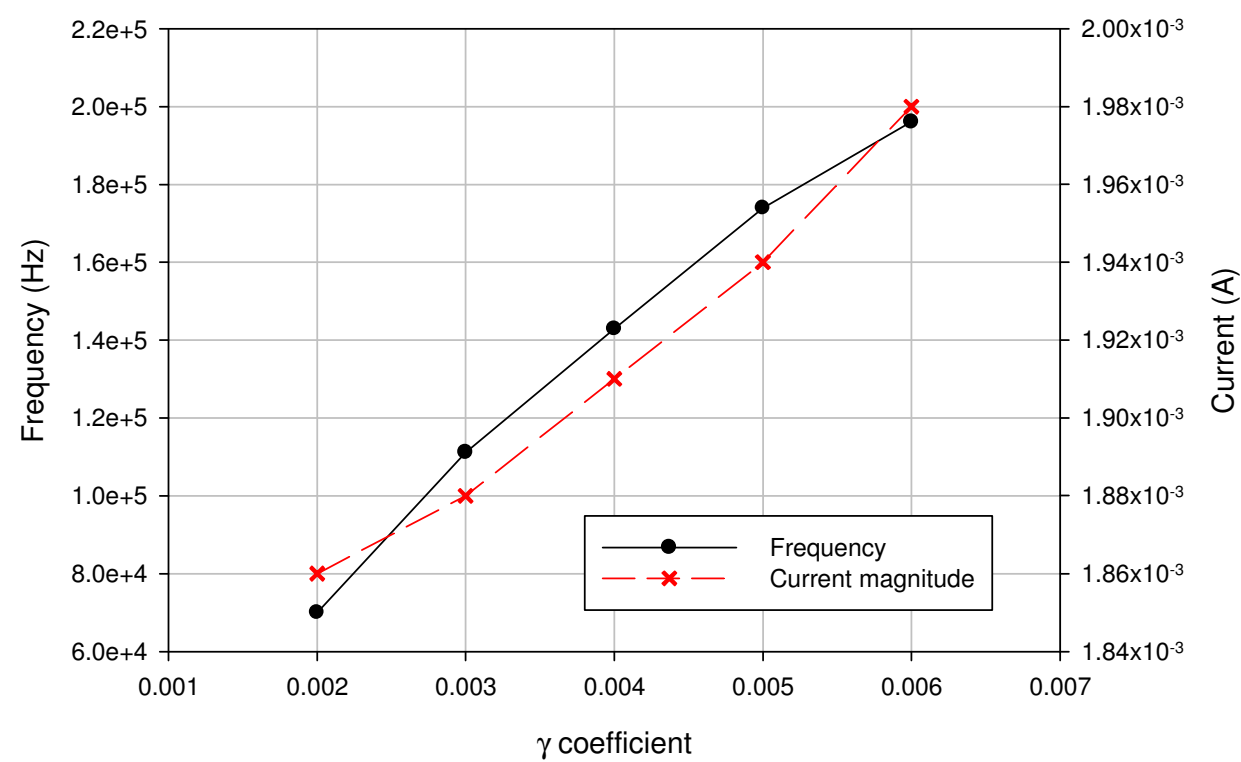

Figure 9. Trichel pulse characteristics predicted for different secondary emission coefficients.

\section{Influence of the applied voltage}

As a validation test for the model, the influence of the applied voltage on the pulse frequency was investigated. According to experimental studies [26], the relationship between the applied voltage and pulse frequency can be expressed as

$$
F=\frac{1}{T}=\frac{K_{1} V\left(V-V_{0}\right)}{r S^{2}}
$$

where $F$ is the frequency $(\mathrm{kHz}), T$ the time period between pulses $(\mathrm{ms}), K_{l}$ a constant depending on the electrode conditions and the surrounding environment, $V$ the applied voltage $(\mathrm{kV}), V_{0}=-2.3(\mathrm{kV})$ the threshold voltage, $r$ the radius of curvature $(\mathrm{mm})$ and $S$ the gap spacing $(\mathrm{mm})$. From this formula, the plot of the frequency $F$ against $V\left(V-V_{0}\right)$ should demonstrate a straight line with a gradient of $K_{l} /\left(r S^{2}\right)$.

The model was run for a range of applied voltage (from -5.4 to $-5.7 \mathrm{kV}$ ) whilst keeping all other parameters constant $(\gamma=0.004)$. Figure 10 shows the dependence of the predicted frequency $F$ on $V\left(V-V_{0}\right)$. The plot clearly shows the linear relationship with a gradient of $38 \mathrm{kHz} \mathrm{kV}$. The increase of the pulse frequency at higher voltages can be understood as follows: as the voltage increases, the electric field is stronger giving rise to a more intensive ionisation and a larger drift velocity. As a consequence the dense plasma forms at shorter times, charged particles are cleared away quicker and the next discharge pulse occurs sooner. 


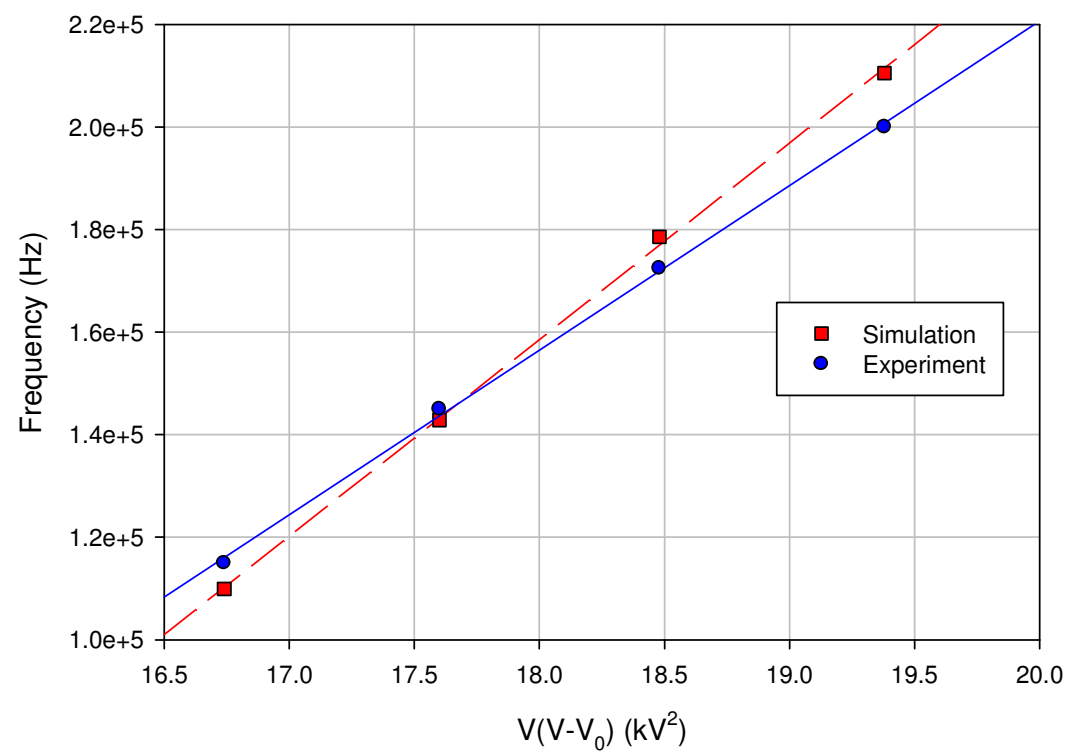

Figure 10. Measured and predicted frequency $F$ as a function of $V\left(V-V_{0}\right)$.

\subsection{Experimental results}

Using the experimental setup described in section 2, the Trichel pulse results obtained at $-5.5 \mathrm{kV}$ applied voltage and $3.3 \mathrm{~mm}$ gap spacing were recorded and shown in figure 11 . The Trichel pulses captured have a frequency of around $144 \mathrm{kHz}$ which is very close to the simulation results obtained with a similar electrode geometry and the secondary emission coefficient set at 0.004. Details of the first experimental current pulse are superimposed onto the simulation result and are shown in figure 11(b). As can be seen, the pulse magnitude is around $3.5 \mathrm{~mA}$ which is higher than the simulation magnitude. Although the rising front of the simulation current waveform is slightly longer than that of the experimental curve, the model seems to predict the right decaying tail. The observed discrepancy is probably due to the influence of the power supply circuitry on the current which is not included into the model. The stochastic appearance of seed electrons in the neighbourhood of the needle and the exclusion of other less important secondary processes may also be accountable. The artificial diffusion used in the model may influence the current magnitude but hardly affect the decaying tail. The injected charge results calculated by integrating the current waveforms are shown in figure 11(c). An excellent agreement between the experimental and simulation injected charge is met. Each current pulse injects approximately $0.11 \mathrm{nC}$ into the system. 


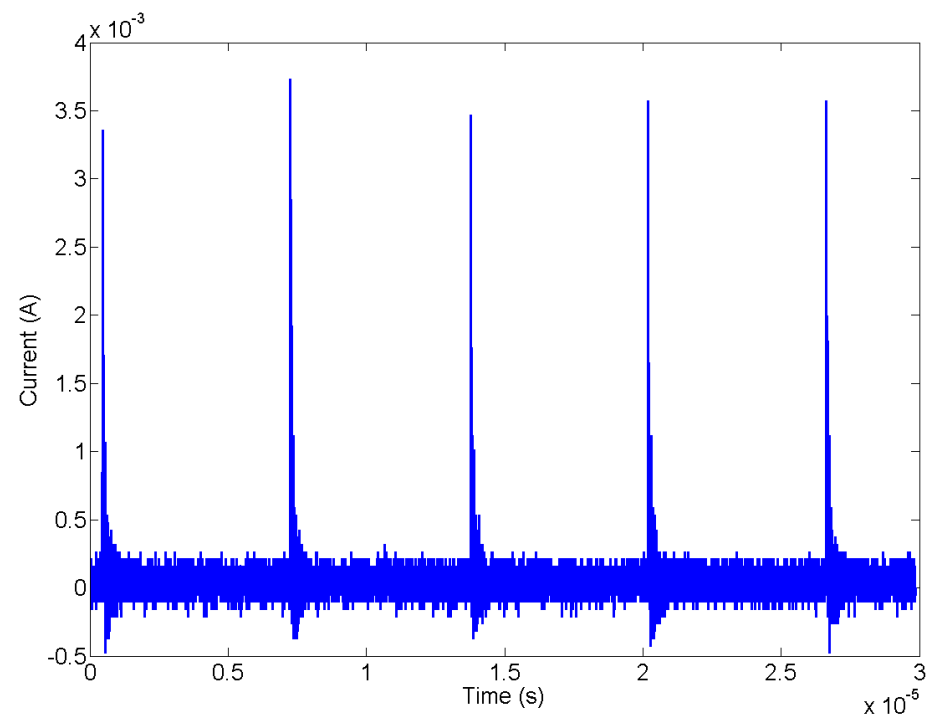

(a) Experimental Trichel pulse sequence

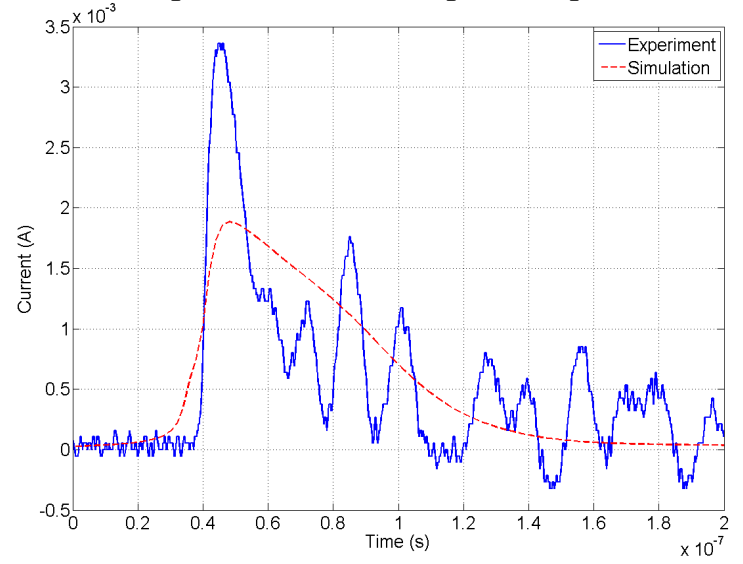

(b) Details of the first current pulse

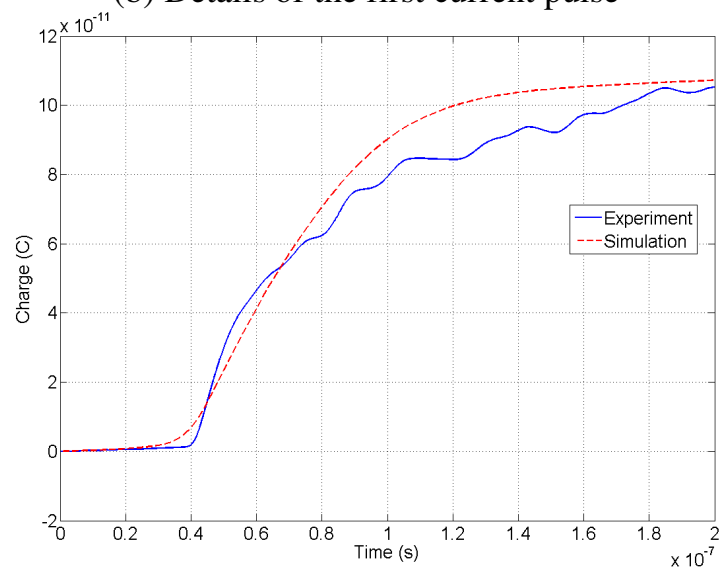

(c) Injected charge waveforms

Figure 11. Trichel pulse results obtained from the experiment and the negative corona discharge model.

In order to verify the empirical relation in equation 9, the experiment was repeated with a range of applied voltage (from -5.4 to $-5.7 \mathrm{kV}$ ) and the corresponding frequency was recorded. Figure 10 shows the observed frequency $F$ against $V\left(V-V_{0}\right)$. As can be seen, the experiment confirmed the linear relationship between the two parameters with a gradient of 
approximately $32 \mathrm{kHz} \mathrm{kV}^{-2}$ and a good agreement between the experimental and simulation results can be observed with a maximum prediction error of $15 \%$.

\section{Negative surface discharge}

Having had the negative corona discharge model validated by comparison with the published data and experiment, the model can be used to solve the problem when dielectric barriers are present. Previous experimental observations of a negative surface discharge suggest that the discharge produces a diffuse and circular/ring distribution of charge [30, 31]. The earlier model proposed in [30] is based on calculations along the strongest field line and can only predict the approximate size of the charge pattern. By modifying the full 2D model presented in section 3, a dynamic simulation of charge accumulation at the dielectric surface can be achieved.

\subsection{Boundary and initial conditions}

In this simulation, the insulation system has been introduced into the gas gap with the details given in figure 3(b). The simulation domain is similar to the corona discharge case with different boundary conditions at dielectric surfaces - boundaries 5-7 in figure 3(b). Most of the initial conditions were kept unchanged from the corona discharge model except the applied voltage which was set to $-2.5 \mathrm{kV}$. Poisson's equation was solved over all 3 subdomains (air gap, BSO and glass) whilst the continuity equations were solved in the gas subdomain only. The same secondary emission coefficient as previously $(\gamma=0.004)$ was used in this model. The upper boundary of the BSO layer was set to free convective flux for electrons and negative ions to let them go through and the fluxes of these charged particles are used to calculate surface charge density at this surface (B5). The flux of positive ions at this boundary, in contrast, was set to zero as it is believed that no positive ions from the dielectric can be released into the gas. The new set of boundary conditions in this model is summarised in table 2 .

Table 2. Boundary conditions for the negative surface discharge model.

\begin{tabular}{|c|c|c|c|c|}
\hline $\begin{array}{l}\text { Application } \\
\text { mode }\end{array}$ & $\begin{array}{c}\text { Convection and } \\
\text { diffusion } \\
N_{e}\end{array}$ & $\begin{array}{c}\text { Convection and } \\
\text { diffusion } \\
N_{p}\end{array}$ & $\begin{array}{c}\text { Convection and } \\
\text { diffusion } \\
N_{n}\end{array}$ & $\begin{array}{c}\text { Electrostatics } \\
V\end{array}$ \\
\hline $\begin{array}{c}\text { Boundaries } \\
1,2,3 \& 4\end{array}$ & $\begin{array}{c}\text { The same as in } \\
\text { Table } 1\end{array}$ & $\begin{array}{c}\text { The same as in } \\
\text { Table } 1\end{array}$ & $\begin{array}{c}\text { The same as in } \\
\text { Table } 1\end{array}$ & $\begin{array}{l}\text { The same as in } \\
\text { Table } 1 \text { except } \\
\text { Potential } \\
V=-2500(\mathrm{~V}) \\
\text { for boundary } 2\end{array}$ \\
\hline Boundary 5 & $\begin{array}{l}\text { Convective flux } \\
\vec{n} \cdot\left(-D_{e} \nabla N_{e}\right)=0\end{array}$ & $\begin{array}{l}\text { Insulation/ } \\
\text { Symmetry } \\
\vec{n} \cdot\left(\overrightarrow{W_{p}} N_{p}\right. \\
\left.-D_{p} \nabla N_{p}\right)=0\end{array}$ & $\begin{array}{l}\text { Convective flux } \\
\vec{n} \cdot\left(-D_{n} \nabla N_{n}\right) \\
=0\end{array}$ & $\begin{array}{l}\text { Surface charge } \\
\vec{n} \cdot\left(\overrightarrow{D_{1}}-\overrightarrow{D_{2}}\right) \\
\quad=\rho_{s}\end{array}$ \\
\hline Boundary 6 & - & - & - & $\vec{n} \cdot\left(\overrightarrow{D_{1}}-\overrightarrow{D_{2}}\right)=0$ \\
\hline Boundary 7 & - & - & - & $\begin{array}{c}\text { Ground } \\
V=0\end{array}$ \\
\hline
\end{tabular}

The field in the gas gap is enhanced by the presence of the insulators and a much lower voltage in comparison with the corona discharge model is needed to be applied at the needle electrode. During the model implementation it has been found that a much finer mesh than that used in the corona discharge case is required in order to obtain a stable algorithm. The mesh size in the region close to cathode was hence reduced to $0.5 \mu \mathrm{m}$. 


\subsection{Simulation results}

With such a gap spacing and electric field, the dielectric barrier discharge undergoes similar discharge events as in the corona discharge case. The significant difference is that only one discharge current pulse forms in this case. The predicted current waveform is shown in figure 12. The pulse is characterised by a fast rising front and a slower decaying tail. On the decaying curve, there exist some oscillations. The current magnitude is just above $1.7 \mathrm{~mA}$, the initial pulse width is around $100 \mathrm{~ns}$ and the tail lasts approximately $1 \mu \mathrm{s}$.

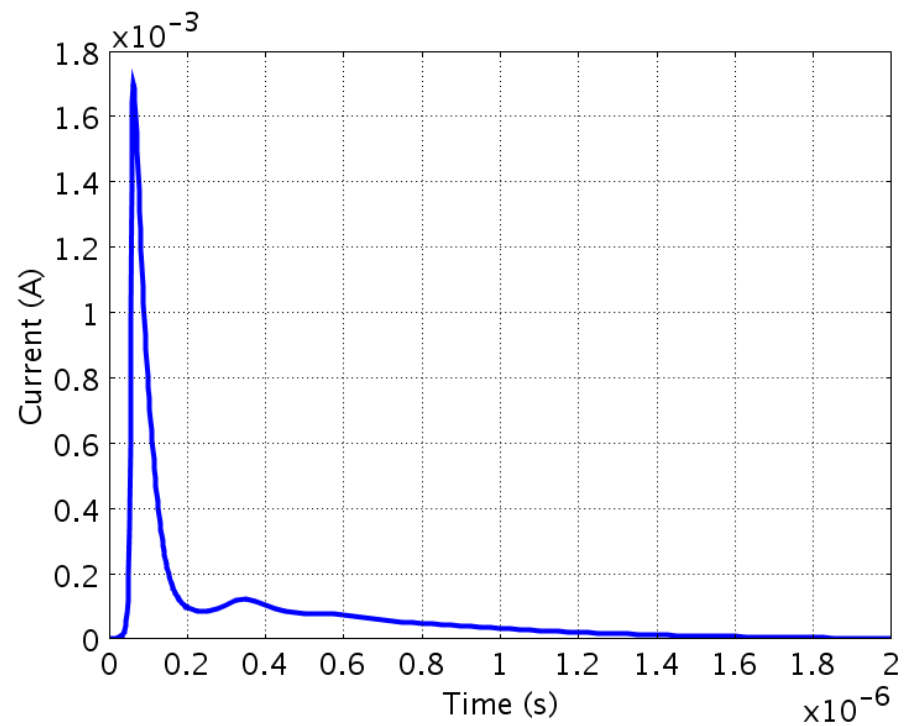

Figure 12. Predicted discharge current from the negative surface discharge model.

Figures 13, 14 and 15 respectively show the 2D charged particle density, electric field and surface charge density distributions at selected times. Similar to the previous analysis in section 4, the Electron Multiplication phase $(0-50 \mathrm{~ns})$ is characterised by the low concentration of positive ions in between the needle electrode and a lower concentration of electrons, figure 13(a). The initial field distribution is not distorted during this phase. Figure 14(a) clearly demonstrates the undisturbed equipotential lines in the gas gap. The surface charge accumulation during this phase is diminutive (figure 15, curve $50 \mathrm{~ns}$ ). This phase is then followed by the Plasma Sheath Formation period (50-62 ns) during which the positive and electron densities increase in magnitude but decrease in size, figure 13(b). Both charged species move towards the cathode as a consequence of the field distortion, figure 14(b). The field distribution plot indicates an increase in magnitude of the field near the needle tip and an increase of the field in the bulk of the insulators due to the surface charge. At the instant the current waveform reaches its peak (62 ns), the surface charge density has a peak value of $0.05 \mathrm{nCmm}^{-2}$, figure 15 .

The Decay process (62-2000 ns) starts after the current has reached its peak. During this phase, positive ion and electron densities decrease but negative ion density increases in magnitude. These negatively charged particles are then swept away towards the dielectric surface due to the electric field. At the beginning of the Decay phase, the increase rate of surface charge density is the largest (figure 15, curves 62-500 ns). This is a result of the movement of the dense cloud of the negative ions, which has a high density just after the Plasma Sheath Formation stage. At the end of the Decay stage, the surface charge density seems to reach its saturation level. The increase rate is much smaller than initially and after $2 \mu$ s the charge density acquires its peak at around $0.37 \mathrm{nCmm}^{-2}$. During the Decay process, the surface charge not only increases in magnitude but also expands radially. This can be explained by the effects of the surface charge field. Initially the charge deposits inside a circle of $\sim 0.22 \mathrm{~mm}$ radius, figure 15 (curve $50 \mathrm{~ns}$ ). As the dielectric surface gradually accumulates the negative charge and the charge density has reached a certain level, the surface charge field tends to oppose the Poisson's field and pushes the incoming ions away from the symmetry 
axis. At the end of the current pulse, the charge accumulates over a ring of $\sim 0.7 \mathrm{~mm}$ radius. The drop of charge density at the centre is evident since the beginning of the Plasma Sheath Formation phase. The feature is linked with the dense plasma position being slightly offset from the symmetry axis, figure 13(b). It is worth noting here that this argument would not be applicable to the positive discharge as in that case electrons are produced at the streamer head by photoionisation.

Effort has been made to investigate the origin of such a ring-shaped density distribution since in the case of positive streamer with photoionisation as the driving mechanism these off-axis structures were attributed to numerical instabilities [32]. This effect was believed to originate from the incorrect representation of 3D phenomena in 2D cylindrical symmetry. To verify the proposed algorithm, space mesh refinement has been performed and smaller time steps have also been examined. The mesh refinement indicated the convergence of the algorithm. In addition, the artificial diffusion coefficient was varied significantly but the results hardly changed. Also a uniformly distributed initial distribution, instead of the Gaussian condition (equation 9) for positive ions and electrons was used and the off-axis behaviour was still observed. Effects of boundary conditions have also been analysed. The boundary condition (equation 6) was artificially modified by including the tangential component of ion outlet velocity. It was shown that the algorithm is not sensitive to the boundary condition implementation. In addition, the negative corona model has been modified to negative plane-plane geometry (infinite radius of curvature needle) to analyse effects of geometry on the observed off-axis behaviour. The off-axis effect was not present in this plane-plane test. As a result, it is quite possible that the observed behaviour may be a genuine phenomenon and it should be subjected to further discussions in the research community.

At the end of the current pulse (2 $\mu$ s - figure 14(c)), the field lines in the air gap tend to return to the Laplacian distribution while the bulk field is still heavily distorted by surface charge. This field distortion appears in the region where the electrons form during the Electron Multiplication phase and this distortion prevents the development of subsequent Trichel pulses. The next pulse can be generated only after the surface charge has dissipated from the BSO. The Pockels experiment suggests that, in general, it takes 1-1.5 seconds for surface charge to decay.
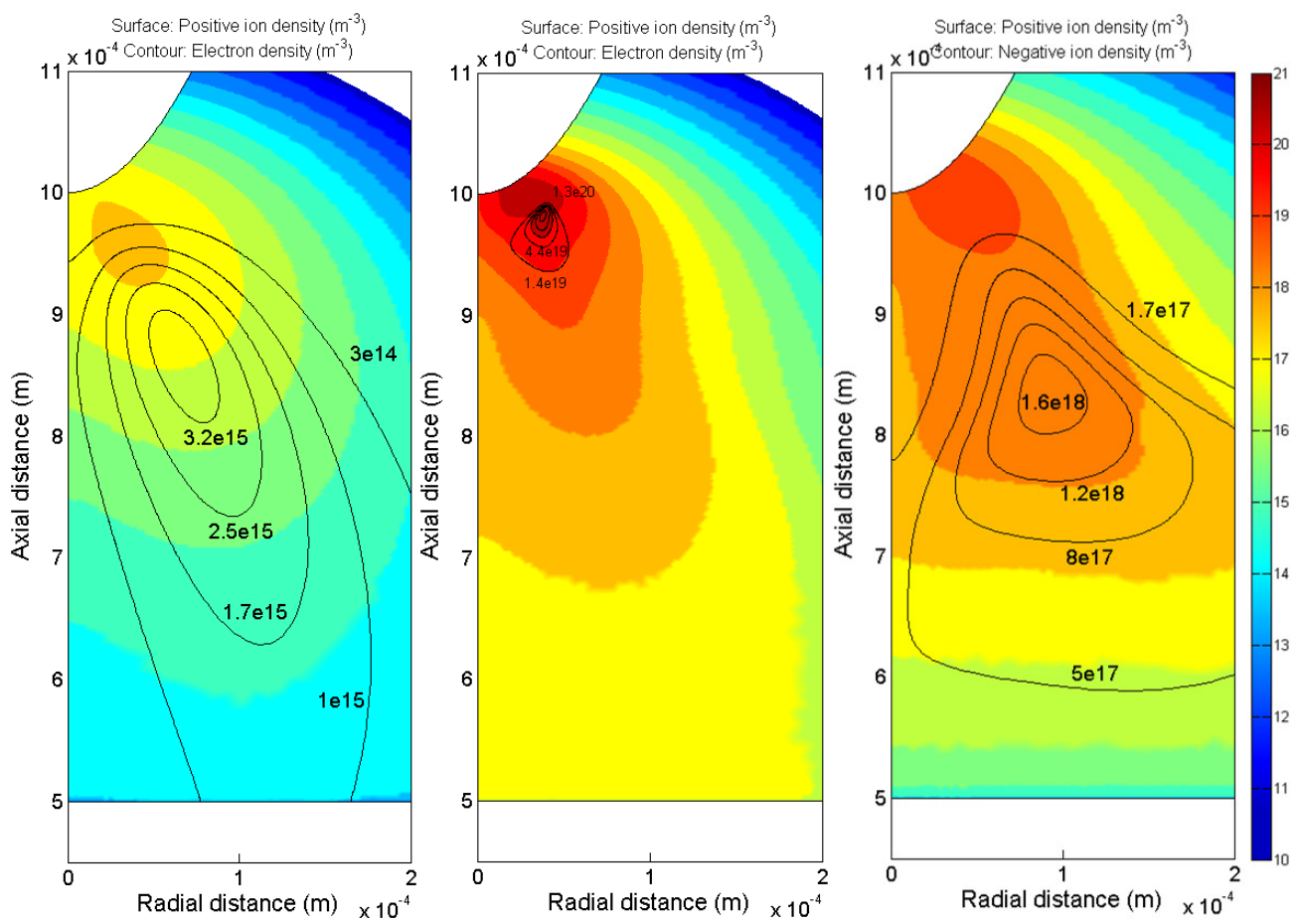

(a) $20 \mathrm{~ns}$
(b) $62 \mathrm{~ns}$
(c) $1000 \mathrm{~ns}$

Figure 13. Predicted 2D distribution of charged particles densities at critical times for the dielectric barrier discharge. Surface plots are in log scale and contour plots are in linear scale.
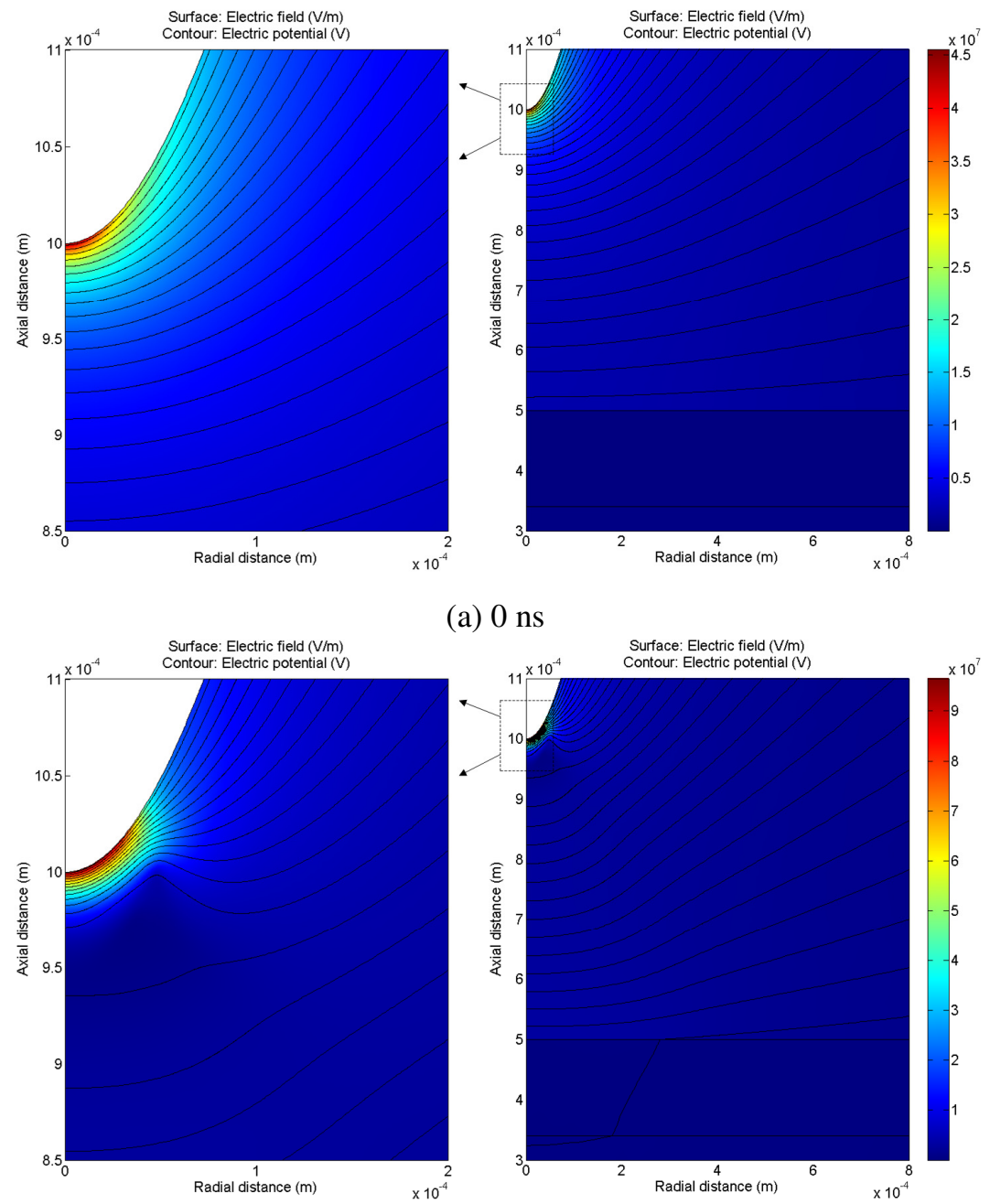

(a) $0 \mathrm{~ns}$

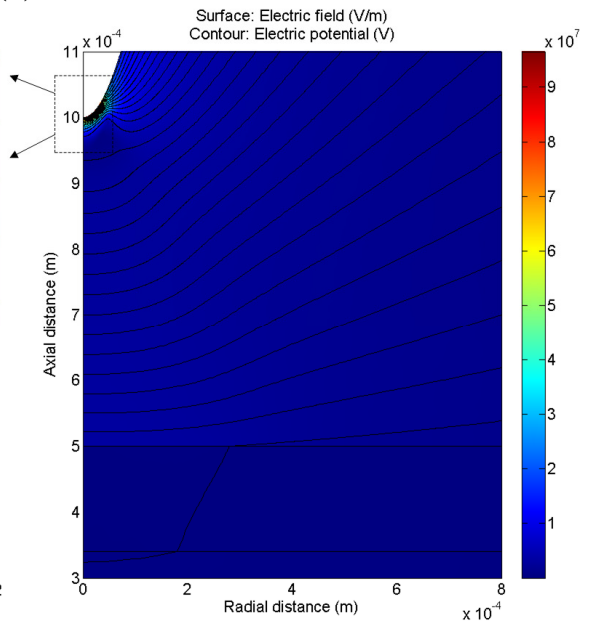

(b) $62 \mathrm{~ns}$
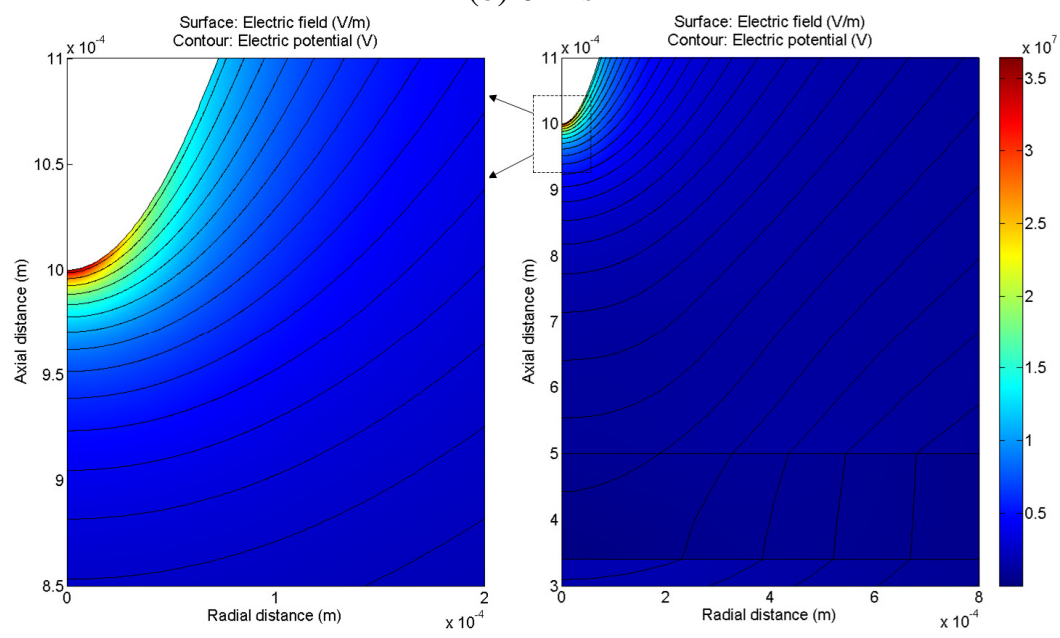

(c) $2000 \mathrm{~ns}$ 
Figure 14. Predicted 2D electric field distribution at critical times for the dielectric barrier discharge.

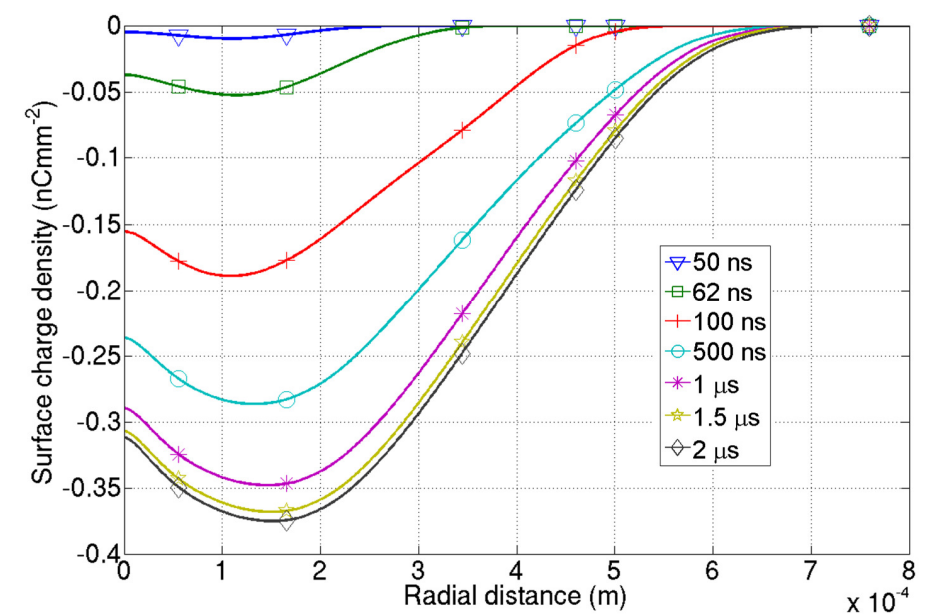

Figure 15. Predicted surface charge density distribution over time.

Influence of the ion-impact secondary emission coefficient

In the case of the negative corona discharge (section 4), the Trichel pulse frequency and the first pulse magnitude linearly increase with the ion-impact secondary emission coefficient. As there is only one discharge pulse for the barrier discharge, this part focuses only on the effects of $\gamma$ coefficient on the pulse magnitude, the surface charge density distribution and the total surface charge (integration of charge density over the dielectric surface). In comparison with the corona discharge case, in this part a wider range of secondary emission coefficients is considered. Figure 16 shows the changes in the peak current magnitude and total surface charge as $\gamma$ varies from 0.002 to 0.012 . It is evident that the current magnitude increases linearly with $\gamma$ and the variations in the current magnitude are much larger than those calculated for the corona discharge (figure 9). The total surface charge also increases but seems to reach a saturation level as $\gamma$ reaches the high values. The charge density distribution at the end of the discharge current pulse calculated for these values of $\gamma$ is shown in figure 17. Generally, an increase in $\gamma$ value results in a rise in charge density and a small radial expansion. As $\gamma$ increases, more charged particles are produced near the cathode and these particles will move towards the dielectric surface at the end of the current pulse. The charge density peak seems to move nearer to the symmetry axis as $\gamma$ increases. At high values of $\gamma$, the increases in the charge density seem to drop due to the field suppression created by the surface charge. Excessive injection of electrons from the needle reduces the total field but does not result in additional charge accumulation above a particular threshold. 


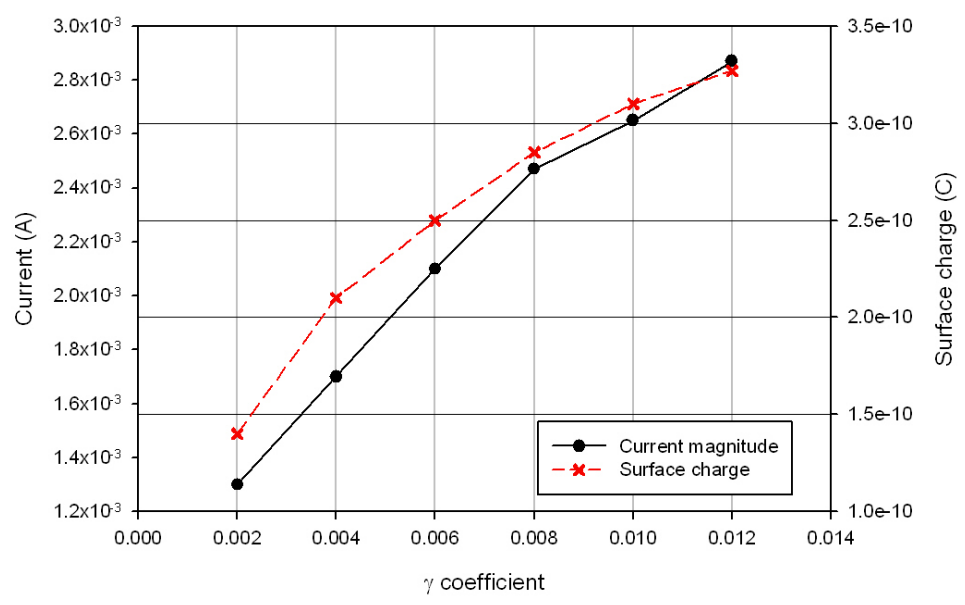

Figure 16. Predicted discharge current pulse magnitude and total surface charge against $\gamma$.

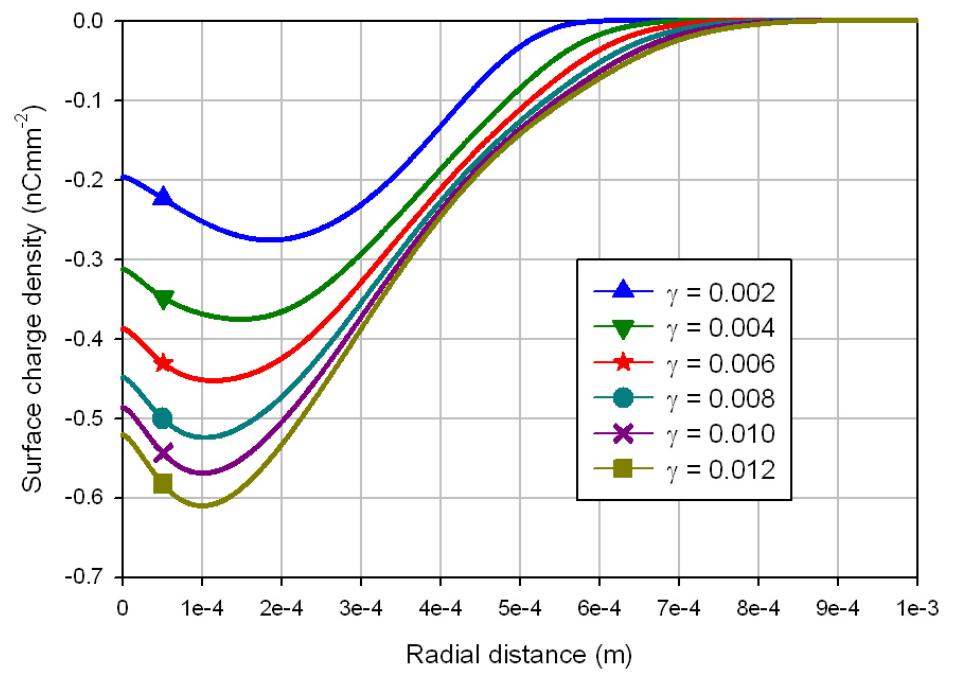

Figure 17. Predicted surface charge density obtained from different secondary emission coefficients.

\subsection{Experimental results}

The surface charge density distribution generated from a negative $2.5 \mathrm{kV}$ square voltage waveform was measured using the Pockels technique and is shown in figure 18. The discharge current waveform, figure 18(a), demonstrates a current pulse of $35 \mathrm{~mA}$ magnitude, initial pulse width of around $10 \mathrm{~ns}$ and the tail of approximately $70 \mathrm{~ns}$ duration. Although these numbers are different from the simulation results, the injected charge seems to be of the same order. The measurement indicates an amount of $0.4 \mathrm{nC}$ charge injected into the system compared with $0.21 \mathrm{nC}$ from the simulation. The experiment suggests 20 times shorter duration but also 20 times stronger current magnitude than predicted. The long tail in the predicted current waveform may stem from the artificial numerical diffusion (section 3.4) but there is another possible source of uncertainty. The electric field is calculated by quasi-static Poisson's equation and effects of the displacement current are neglected. Since the duration of the real discharge is quite short and the BSO has a high permittivity the quasi-static approximation could bring significant errors.

The measured two-dimensional charge density distribution and a typical radial charge density are shown in figures 18 (b) and (c). The charge is distributed over the diameter of 
around $2.1 \mathrm{~mm}$ with the peak charge density of $0.6 \mathrm{nCmm}^{-2}$. A drop in the charge density distribution at the symmetry axis position is noticeable. The predicted radius of the injected charge region is $30 \%$ less than that measured experimentally. The agreement is better for higher $\gamma$ values $(\sim 0.01)$. Taking into account the uncertainties in $\gamma$ and differences in the experimental and modelled needle shapes it can be concluded that the set of equations 1-4 could be effectively used to analyse dielectric barrier discharges.

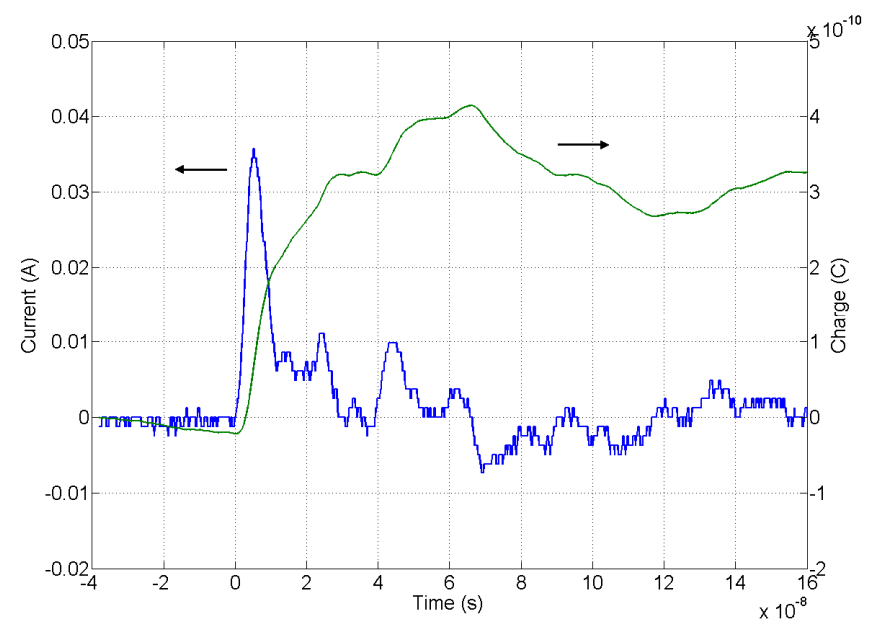

(a) Current and injected charge waveforms

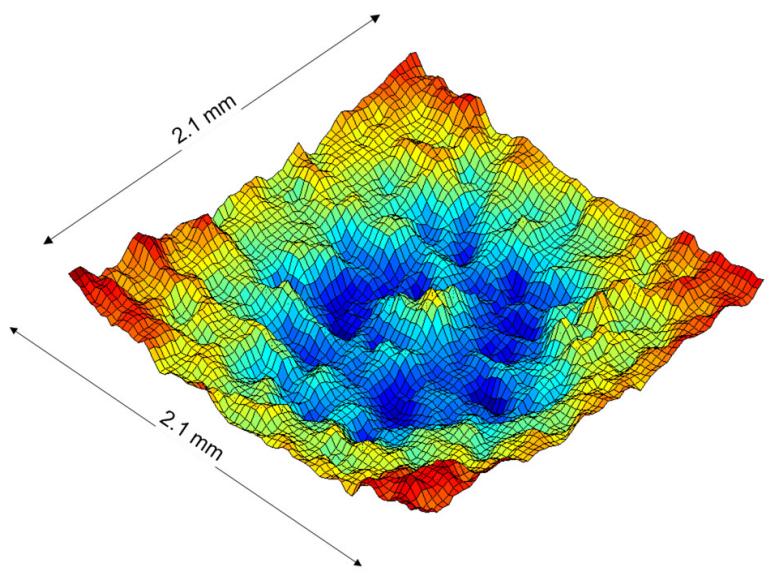

(b) 2D surface charge density distribution 


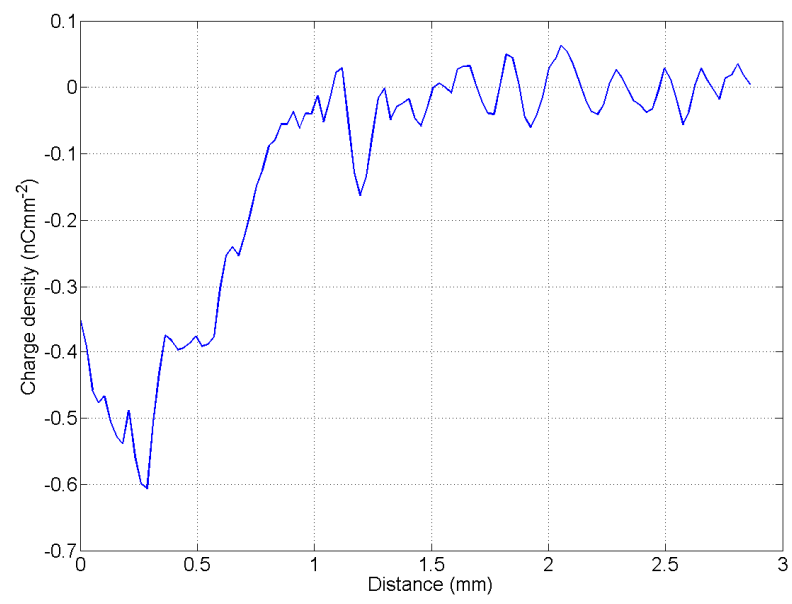

(c) Radial charge density distribution

Figure 18. Experimental negative surface discharge results.

\section{Conclusion}

Negative discharges in air without and with dielectrics were analysed by $2 \mathrm{D}$ axisymmetric finite element models. The model input parameters were verified by experiments. It was shown that the model successfully describes both the localised nature of the discharges and their global characteristics. The observations can be summarised as follows:

- At the Electron Multiplication stage of the discharge the peaks of both positive ion and electron densities are not on the symmetry axis but situated at some distance away from the axis and the dense plasma actually forms a charged torus ring rather than a charged ellipsoid. Possible effects of numerical instabilities and implementation of boundary conditions were investigated in detail. It was concluded that the algorithm is stable and converges as the mesh size and time steps are reduced. It was suggested that the observed behaviour could be a result of imposed 2D axial symmetry which might not accurately describe the whole problem and full 3D modelling is needed to achieve perfect results. Nevertheless even 2D studies allow describing key features of the dielectric barrier discharges observed experimentally.

- It results in ring-like charge distribution on the surface of the dielectric barrier with a noticeable drop in the charge density at the symmetry axis position. Although such behaviour looks artificial, the charge distribution measured by the Pockels technique supports arguments for the observed off-axis effect. The problem should be subjected to further discussions in the research community.

- Subsequent pulses in the Trichel sequence appear when the plasma particles are cleared away from the air gap and the field returns to its initial distribution.

- In the case of the dielectric barrier only one discharge current pulse is produced instead of a pulse sequence as for the corona discharge case. The accumulated surface charge distorts the electric field, depresses electrons multiplication and prevents the formation of subsequent pulses.

- It was found that the model predictions and experimental results agree well when the ion-impact secondary emission coefficient $\gamma=0.004$ is used.

- The magnitude of the current pulse depends linearly on $\gamma$ but at high values of $\gamma$ the total surface charge reaches a saturation level. 


\section{References:}

[1] Georghiou G E, Papadakis A P, Morrow R and Metaxas A C 2005 Numerical modelling at atmospheric pressure leading to plasma production J. Phys. D: Appl. Phys. 38 303-28

[2] Kogelschatz U 2002 Dielectric-barrier discharges: their history, discharge physics, and industrial applications Plasma Chemistry and Plasma Processing 23 1-46

[3] Penetrante B M and Schultheis S E 1993 Non-thermal plasma techniques for pollution control; Parts A and B (NATO ASI) (Berlin: Springer)

[4] Stoffels E, Flikweert A J, Stoffels W W and Kroesen G M W 2002 Plasma needle: a non-destructive atmospheric plasma source for fine surface treatment of (bio)materials Plasma Sources Science Technology 11 383-8

[5] Georghiou G E, Morrow R and Metaxas A C 1999 Characterisation of point-plane corona in air at radio frequency using a FE-FCT method J. Phys. D: Appl. Phys. 32 2204-18

[6] Lagmich Y, Callegari T, Pitchford L and Boeuf J 2008 Model description of surface dielectric barrier discharges for flow control J. Phys. D: Appl. Phys. 41

[7] Murooka Y, Takada T and Hidaka K 2001 Nanosecond surface discharge and charge density evaluation, part i: review and experiments IEEE Electrical Insulation Magazine 17(2) 6-16

[8] Trichel G W 1938 The mechanism of the negative point to plane corona near onset Physical Review 54 1078-84

[9] Morrow R 1985 Theory of negative corona in oxygen Physical Review A 321799-1809

[10] Espel P, Paillol J, Reess T, Gilbert A and Domens P 2001 Numerical modelling of negative corona discharge in $\mathrm{SF}_{6}$ at atmophseric presure J. Phys. D: Appl. Phys. 35 $318-27$

[11] Soria-Hoyo C, Pontiga F and Castellanos A 2007 Particle-in-cell simulation of Trichel pulses in pure oxygen J. Phys. D: Appl. Phys. $404552-60$

[12] Sam Y L, Lewin P L, Davies A E, Wilkinson J S, Sutton S J and Swingler S G 2003 Surface discharge measurements of polymeric materials IEE Proceedings: Science, Measurement and Technology 150 43-52

[13] Kang W S, Park J M, Kim Y and Hong S H 2003 Numerical study on influences of barrier arrangements on dielectric barrier discharge characteristics IEEE Trans. Plasma. Sci. 31 504-10

[14] Georghiou G E, Morrow R and Metaxas A C The theory of short-gap breakdown of needle point-plane gaps in air using finite-difference and finite-element methods $J$. Phys. D: Appl. Phys. 32(12) 1370-1385

[15] Gupta D K, Mahajan S and John P I 2000 Theory of step on leading edge of negative corona current pulse J. Phys. D: Appl. Phys. 33 681-91

[16] Georghiou G E, Morrow R and Metaxas A C 2001 The effect of photoemission on the streamer development and propagation in short uniform gaps J. Phys. D: Appl. Phys. 34 200-8

[17] O'Sullivan F M 2007 A model for the initiation and propagation of electrical streamers in transformer oil and transformer oil based nanofluids $P h D$ thesis, MIT

[18] Morrow R and Sato N 1999 The discharge current induced by the motion of charged particles in time-dependent electric fields; Sato's equation extended J. Phys. D: Appl. Phys. 30 L20

[19] Sato N 1980 Discharge current induced by the motion of charged particles J. Phys. D: Appl. Phys. 13 L3-6

[20] COMSOL Inc., "COMSOL Multiphysics simulation environment. Version 3.5a manual". 2009, Stockholm, Sweden

[21] Zienkiewicz O, Taylor R and Nithiarasu P 2005 The finite element method for fluid dynamics Elsevier $6^{\text {th }}$ edition

[22] Codina R 1998 Comparison of some finite element methods for solving the diffusionconvection-reaction equation Computer Methods in Applied Mechanics and 
Engineering 156 185-210

[23] Lee S H, Lee S Y, Chung Y K and Park I H 2007 Finite-element analysis of corona discharge onset in air with artificial diffusion scheme and under Fowler-Nordheim electron emission IEEE Transactions on Magnetics 43 1453-6

[24] Georghiou G E, Morrow R and Metaxas A C 1999 An improved finite-element fluxcorrected transport algorithm Journal of Computational Physics 148 605-20

[25] Gear C 1971 Numerical initial value problems in ordinary differential equations Prentice Hall, Englewood Cliffs, NJ, USA

[26] Lama W L and Gallo C F 1974 Systematic study of the electrical characteristics of the "Trichel" current pulses from negative needle-to-plane coronas Journal of Applied Physics 45 103-13

[27] Akishev Y S, Kochetov I V, Loboiko A I and Napartovich A P 2002 Numerical simulations of Trichel pulses in a negative corona in air Plasma Physics Reports $\mathbf{2 8}$ 1049-59

[28] Tran T N, Golosnoy I O, Lewin P L and Georghiou G E 2009 Two dimensional studies of Trichel pulses in air using the finite element method IEEE Conference on Electrical Insulation and Dielectric Phenomena, Virginia Beach, USA

[29] Cernak M, Hosokawa T, Odrobina I 1993 Experimental confirmation of positivestreamer-like mechanism for negative corona current pulse rise J. Phys. D: Appl. Phys. 26 607-18

[30] Manabe Y and Shimazaki T 2004 Formation mechanism of surface corona on dielectric plates under negative impulse voltage in atmospheirc air IEEE Transactions on Dielectrics and Electrical Insulation 11(4) 631-37

[31] Celestin S, Allegraud K, Canes-Boussard G, Leick N, Guaitella O and Rousseau A 2008 Patterns of plasma filaments propagating on a dielectric surface IEEE Transactions on Plasma Science 36(4) 1326-27

[32] S Pancheshnyi and A Starikovskii 2001 Comment on "The role of photoionization in positive streamer dynamics" J. Phys. D: Appl. Phys. 34 248-50 